\title{
Characterizing Material Property Tradeoffs of Polycrystalline Diamond for Design Evaluation and Selection
}

Neil David Haddock

Brigham Young University - Provo

Follow this and additional works at: https://scholarsarchive.byu.edu/etd

Part of the Mechanical Engineering Commons

\section{BYU ScholarsArchive Citation}

Haddock, Neil David, "Characterizing Material Property Tradeoffs of Polycrystalline Diamond for Design Evaluation and Selection" (2009). Theses and Dissertations. 1808.

https://scholarsarchive.byu.edu/etd/1808

This Thesis is brought to you for free and open access by BYU ScholarsArchive. It has been accepted for inclusion in Theses and Dissertations by an authorized administrator of BYU ScholarsArchive. For more information, please contact scholarsarchive@byu.edu, ellen_amatangelo@byu.edu. 


\section{CHARACTERIZING MATERIAL PROPERTY TRADEOFFS OF POLYCRYSTALLINE DIAMOND FOR DESIGN SELECTION AND EVALUATION}

by

Neil D. Haddock

A thesis submitted to the faculty of

Brigham Young University

in partial fulfillment of the requirements for the degree of

Master of Science

Department of Mechanical Engineering

Brigham Young University

August 2009 

Copyright (C) 2009 Neil D. Haddock

All Rights Reserved 

BRIGHAM YOUNG UNIVERSITY

GRADUATE COMMITTEE APPROVAL

of a thesis submitted by

Neil D. Haddock

This thesis has been read by each member of the following graduate committee and by majority vote has been found to be satisfactory.

Date

Date

Date
Christopher A. Mattson, Chair

Kenneth W. Chase

David T. Fullwood 



\section{BRIGHAM YOUNG UNIVERSITY}

As chair of the candidate's graduate committee, I have read the thesis of Neil D. Haddock in its final form and have found that (1) its format, citations, and bibliographical style are consistent and acceptable and fulfill university and department style requirements; (2) its illustrative materials including figures, tables, and charts are in place; and (3) the final manuscript is satisfactory to the graduate committee and is ready for submission to the university library.

Date

Accepted for the Department
Christopher A. Mattson

Chair, Graduate Committee
Larry L. Howell

Graduate Coordinator

Accepted for the College

Alan R. Parkinson

Dean, Ira A. Fulton College of Engineering and Technology 



\begin{abstract}
CHARACTERIZING MATERIAL PROPERTY TRADEOFFS OF POLYCRYSTALLINE DIAMOND FOR DESIGN SELECTION AND EVALUATION
\end{abstract}

\author{
Neil D. Haddock \\ Department of Mechanical Engineering \\ Master of Science
}

Polycrystalline diamond (PCD) is used as a cutting tool in many industries because of its superior wear resistance compared to single crystal diamond. Engineers who design new PCD materials must have an understanding of the tradeoffs between material properties in order to tailor a product for different applications. Two competing material properties that are often encountered in PCD are transverse rupture strength and thermal-resistance. Thermal-resistance is directly related to the cobalt content of PCD, and is the ability of the material to withstand thermally induced degradation.

In this thesis, we characterize the tradeoff boundary between transverse rupture strength and cobalt content of PCD. We also characterize the tradeoff boundary between cost and cobalt content, and show how both of these tradeoff boundaries can be used to 

manage product development, which adds value for managers in both engineering and business.

In order to characterize these tradeoffs, empirical models are developed for each material property in terms of the design variables of sintering pressure and diamond grain size, where the pressure ranges from $55 \mathrm{kbar}$ to $77 \mathrm{kbar}$ and the grain size ranges from 12 $\mu \mathrm{m}$ to $70 \mu \mathrm{m}$ in diameter. Then the models are used as optimization objectives in the normal constraint method to generate the tradeoff boundary. Finally, the tradeoff boundary is validated through additional experiments.

The tradeoff boundary shows that the relationship between transverse rupture strength and cobalt content is not linear. It also shows that the optimal PCD designs can occur over a wide range of pressures and grain sizes, but pressures above $66 \mathrm{kbar}$ and grain sizes between 20 and $30 \mu \mathrm{m}$ appear to offer the best compromise between these material properties. These results are compared to the wear rates of PCD compacts in rock cutting tests. The rock cutting test results confirm that the designs with the best compromise between transverse rupture strength and cobalt content also have the highest wear resistance. In general, the designs that offer the best compromise between the properties are also the most expensive to manufacture. 



\section{ACKNOWLEDGMENTS}

There are many people who have not only contributed to the success of this thesis, but also to my success as a graduate student and engineer.

I want to first thank Dr. Chris Mattson for his guidance and support. He helped me find a project that balanced the competing objectives of my career and my education, and pushed me to do things that I did not think were possible. I also extend appreciation to the rest of my committee, Dr. Ken Chase and Dr. David Fullwood, for their support and contributions to this work.

I especially wish to thank the people at US Synthetic who provided the motivation, funding, and valuable insights into the research that has become this thesis. They include Dr. Ken Bertagnolli, Dr. Deb Mukhopadhyay, Craig Cooley, David Miess, Jason Wiggins, Jake Atwater, and the R\&D lab at US Synthetic. I also thank Bob Johnson and Robert Farr for allowing me the time and freedom to pursue my educational goals while working full-time.

I express deep gratitude to my wife, Brynna, for her patience, support, and personal sacrifices during this journey. Her continual encouragement has been invaluable to the completion of this work. And to my mother and father, who taught me by example to make education a daily pursuit.

Last, I express gratitude to the Lord from whom all of my life's blessings come. This thesis is living proof that with Him, all things are possible. 



\section{TABLE OF CONTENTS}

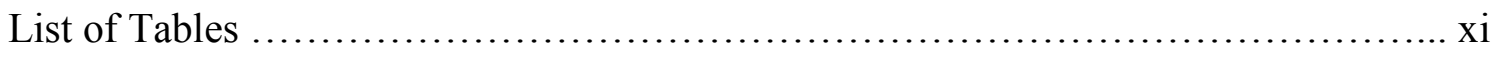

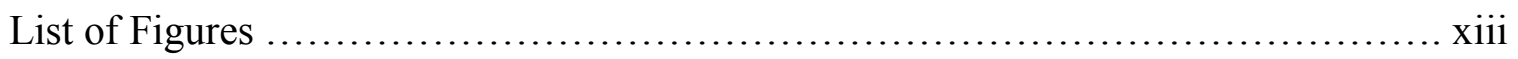

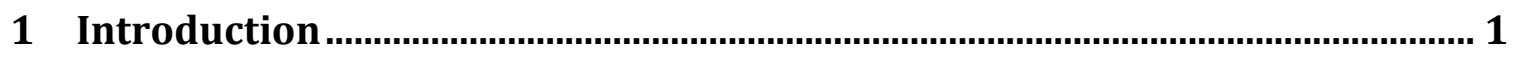

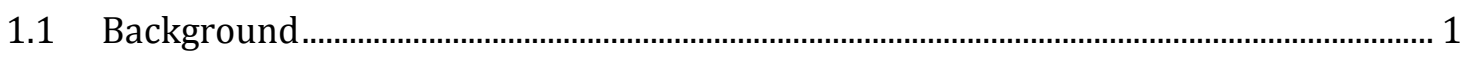

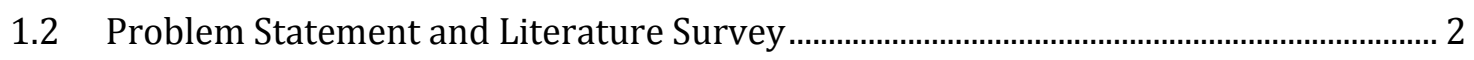

1.3 Research Approach ..................................................................................................

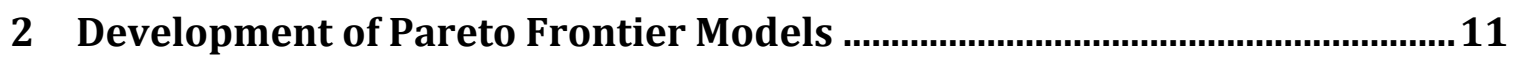

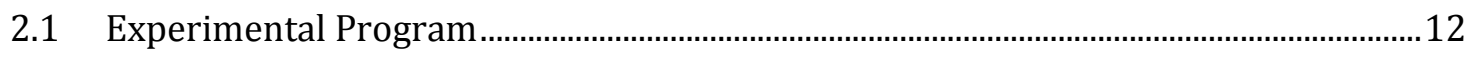

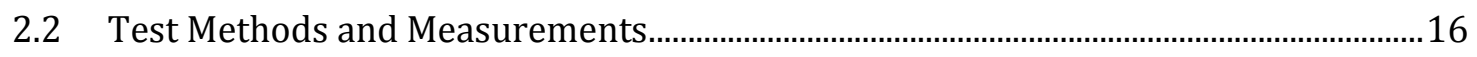

2.3 Response Surface Model for Cobalt Content.........................................................................18

2.4 Response Surface Model for Transverse Rupture Strength ………………………….....19

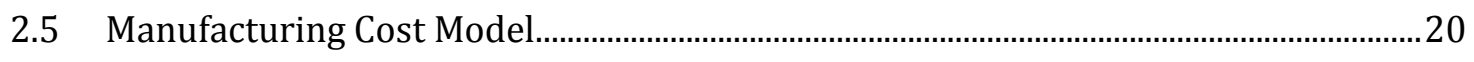

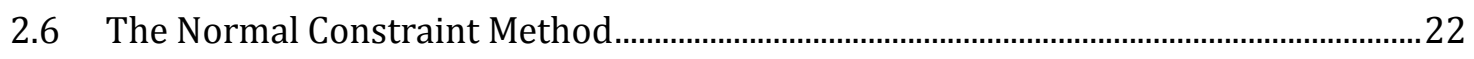

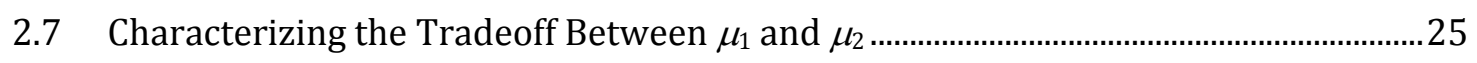

2.7.1 Iteration 1

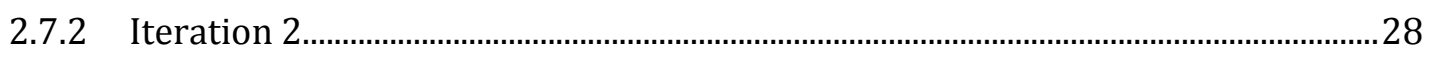

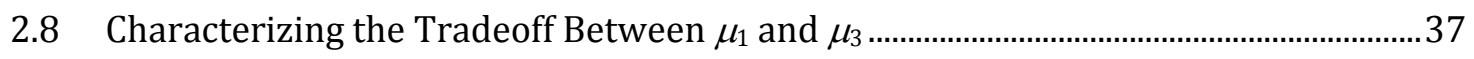

3 Design Evaluation and Selection Using Pareto Frontier Models .....................41

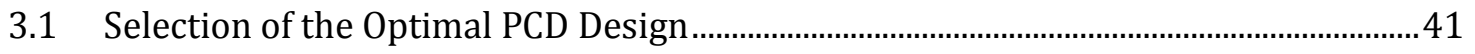


3.2 Evaluation of the Optimal PCD Design ............................................................................. 45

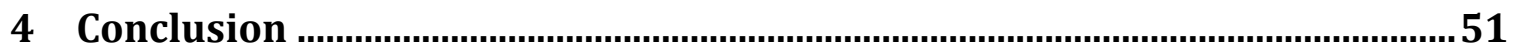

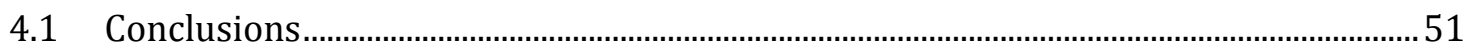

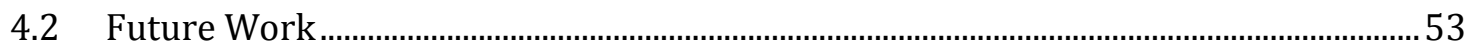

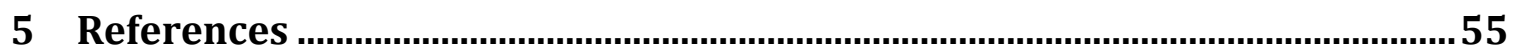

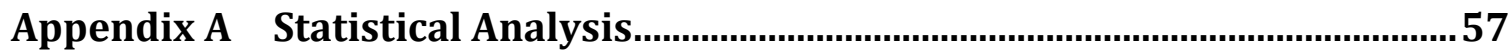

A.1 Normally Distributed Errors with a Zero Mean ................................... 58

A.2 Constant Variance and Independent Errors ................................... 61

A.3 Discussion of Model Assumptions . ....................................... 64 


\section{LIST OF TABLES}

Table 2.1: Design variable values in coded (parenthesis) and uncoded form .....................15

Table 2.2: Summary statistics for experimental runs A - I (10 samples each) ..................16

Table 2.3: Summary of transverse rupture strength measurements .................................17

Table 2.4: Summary of the additional experimental runs, J-M .....................................29

Table 2.5: Output data from Statistica used to obtain equation (2.4) ................................31

Table 3.1: Rock cutting parameters for Test I and Test II..........................................46 


\section{LIST OF FIGURES}

Figure 1.1: A polycrystalline diamond compact (a) with PCD layer (b) sintered onto cemented WC/Co substrate (c) ............................................................................

Figure 1.2: Three-step approach for developing the Pareto frontier model..........................9

Figure 2.1: Pressure-Temperature phase diagram for carbon ..............................................14

Figure 2.2: Experimental runs shown in the design variable space .....................................15

Figure 2.3: Transverse rupture strength test setup ..............................................................17

Figure 2.4: Cobalt content response surface model plotted against the experimental

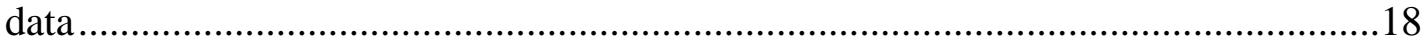

Figure 2.5: Transverse rupture strength response surface model .......................................19

Figure 2.6: Graphical representation of the normal constraint method for bi-objective

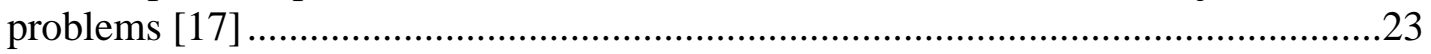

Figure 2.7: The Pareto frontier for $\mu_{1}$ and $\mu_{2}$, generated using equations (2.3) and (2.4)....26

Figure 2.8: The Pareto frontier with a "confidence region" drawn around the 95\% confidence limits on the mean for each segment .......................................................27

Figure 2.9: A comparison of the first Pareto frontier model to the experimental data .........28

Figure 2.10: Contour plot of transverse rupture strength showing the pressure and grain

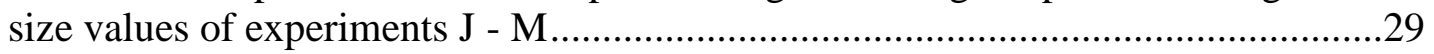

Figure 2.11: Average values compared to quadratic model predictions of $\mu_{2}$ for designs

$\mathrm{C}, \mathrm{D}$ and $\mathrm{J}$

Figure 2.12: Average values of $\mu_{2}$ for designs $\mathrm{C}, \mathrm{D}$ and $\mathrm{J}$ compared to the linear and quadratic model predictions

Figure 2.13: Comparison of the quadratic (a) and linear (b) response surface models for

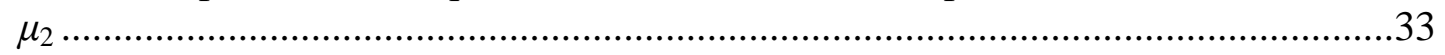

Figure 2.14. Comparison of the first and second Pareto frontier approximations .34 
Figure 2.15: The second and final Pareto frontier model with the 95\% confidence region

Figure 2.16: The first Pareto frontier model plotted against all 13 experimental runs .........35

Figure 2.17: The second Pareto frontier mapped to the design variable space....................36

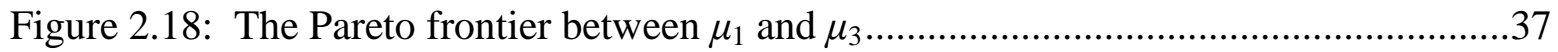

Figure 2.19: The Pareto frontier between $\mu_{1}$ and $\mu_{3}$ plotted against the experimental data..39

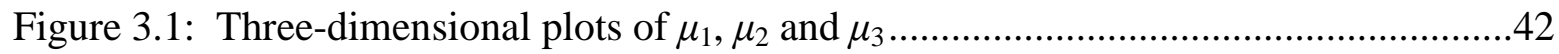

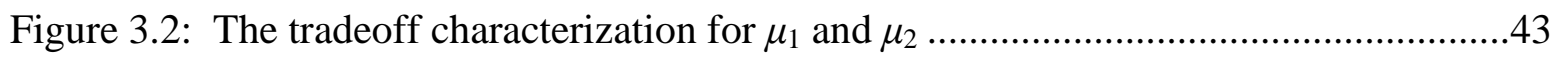

Figure 3.3: Three-dimensional plot of $\mu_{1}, \mu_{2}$ and $\mu_{3}$ showing the most "optimal" PCD

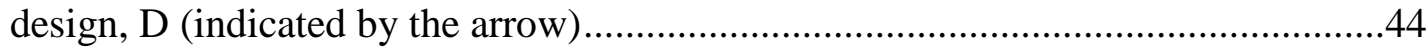

Figure 3.4: Vertical turret Lathe apparatus used for testing the wear rate of PCD.............46

Figure 3.5: Contour plot of the G-ratio for designs A - I (Test I), with Pareto frontier highlighted

Figure 3.6: Contour plot of distance-to-failure for designs A - I (Test II) ........................47

Figure A.1: The normal probability plot for the errors of equation $(2.3)$.........................58

Figure A.2: The normal probability plot for the errors of equation $(2.4)$..........................59

Figure A.3: The normal probability plot for the errors of equation (2.16) .......................59

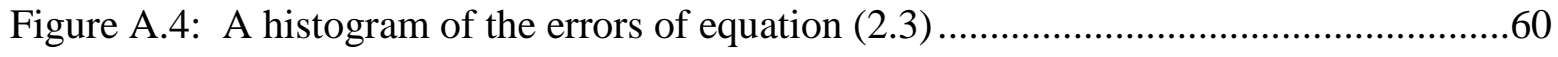

Figure A.5: A histogram of the errors of equation (2.4) ...........................................60

Figure A.6: A histogram of the errors of equation (2.16) showing a similar pattern to the

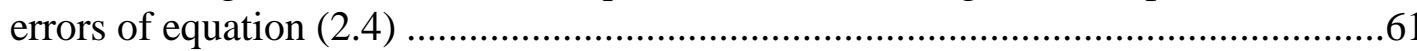

Figure A.7: A plot of the errors (vertical axis) vs. the values predicted by the model (horizontal axis) for equation (2.3)

Figure A.8: A plot of the errors vs. the values predicted by the model for equation (2.4)

Figure A.9: A plot of the errors vs. the values predicted by the model for equation (2.16) 



\section{Introduction}

\subsection{Background}

Engineering design often involves making decisions between two or more conflicting objectives. When designing a new material to meet a given need or selecting an existing material for a particular application, the conflicting objectives often take the form of competing material properties. For example, tensile strength is typically improved to the detriment of fracture toughness, and vice versa [1]. Materials engineers are usually tasked with resolving these types of competing objectives. But, whether a materials engineer seeking to improve a given tradeoff or a design engineer responsible for selecting a suitable material for a given component, having an explicit characterization of material property tradeoffs can enhance the design selection process or provide direction towards materials improvement efforts.

Polycrystalline diamond (PCD) is widely used as a material in cutting tools, wire drawing, rock cutting and other wear surface applications. In order to design better wear tools, engineers must understand the wear and chipping mechanisms of PCD [2]. An understanding of material properties such as fracture toughness, tensile strength, compressive strength, and elastic modulus, and the relationships between these properties, is therefore an important part of PCD design. Another important property of PCD is its ability to resist degradation under thermal loads. This is referred to as thermal stability, or thermal-resistance [3]. Two competing objectives, often encountered in the 
design of PCD tools, are transverse rupture strength - a tensile property [4] - and thermal-resistance.

When a designer faces decisions between competing objectives, it is possible that more than one solution exists that will meet the design goals. Having a complete visual representation of all possible designs in relation to the objectives would aid the designer in making the optimal design decision. It is possible that some designs would be better than others in both objectives, and thus would be the most relevant to the designer. An effective way of characterizing those designs is by finding the Pareto frontier [5]. The Pareto frontier is the set of all non-dominated designs within the feasible design space, which means that no other designs exists that are equal or better in every objective. When a design solution falls on the Pareto frontier we say it is Pareto optimal [6]. With a set of Pareto optimal solutions in hand, a designer can see the "best" options available under the specified constraints [7].

In this thesis, we use multi-objective optimization methods to characterize the tradeoff between the objectives of transverse rupture strength and thermal-resistance of PCD. We also characterize the tradeoff between a manufacturing cost objective and thermal-resistance in order to create value for engineering and business managers. These tradeoffs are characterized as Pareto frontier models. Finally, we compare the Pareto frontier models to the wear rates of PCD in rock cutting tests.

\subsection{Problem Statement and Literature Survey}

Characterizing the design tradeoffs by modeling the Pareto frontier using multiobjective optimization methods requires mathematical representations of the objectives in terms of the design variables. Several authors have measured the transverse rupture 
strength of PCD $[2,10]$, and others have qualitatively described the factors that influence its thermal-resistance $[3,11]$. Before we review this literature however, we will briefly review the fundamentals of polycrystalline diamond manufacturing and the unique challenges associated with testing diamond.

Diamond powder is sintered to form PCD under pressure and temperature conditions where diamond is the thermodynamically stable phase of carbon. These pressure and temperature ranges are approximately $50 \mathrm{kbar}$ and above, and $1500^{\circ} \mathrm{C}$ to $2000^{\circ} \mathrm{C}$, respectively [8]. The diamond is typically sintered onto a cemented tungsten carbide substrate containing a cobalt binder. This substrate provides support for the diamond layer and a means for attaching it to a given tool. Also, under high pressure and high temperature conditions, the cobalt binder alloy from the substrate melts and infiltrates the diamond powder, and facilitates diamond-to-diamond growth by enhancing the bonding kinetics between grains [9]. This sintering process produces a coherent diamond structure with residual cobalt metal left in the pore spaces between diamond grains. Figure 1.1 shows a polycrystalline diamond compact (a) with a sintered diamond layer (b) that is bonded to a cemented tungsten carbide substrate (c). Figure 1.1b illustrates the bonding that occurs between diamond grains (dark areas), and shows residual cobalt left in the structure (light areas).

The ability of engineers to model and predict the behavior of PCD without going to the expense of building and testing many samples is limited. Testing diamond samples is a challenge for several reasons. First, creating the high temperature and high pressure environment necessary for diamond formation requires manufacturing equipment that is costly to obtain and operate. Second, because diamond is the hardest known material, 
specialized fixtures are often required for mechanical testing. A third obstacle in working with diamond is the difficulty one has in forming it into standard test sizes and geometries. Finally, the repeatability of tensile strength measurements is difficult to achieve. These challenges can make the gathering of material property data for PCD more difficult compared to other engineering ceramics.

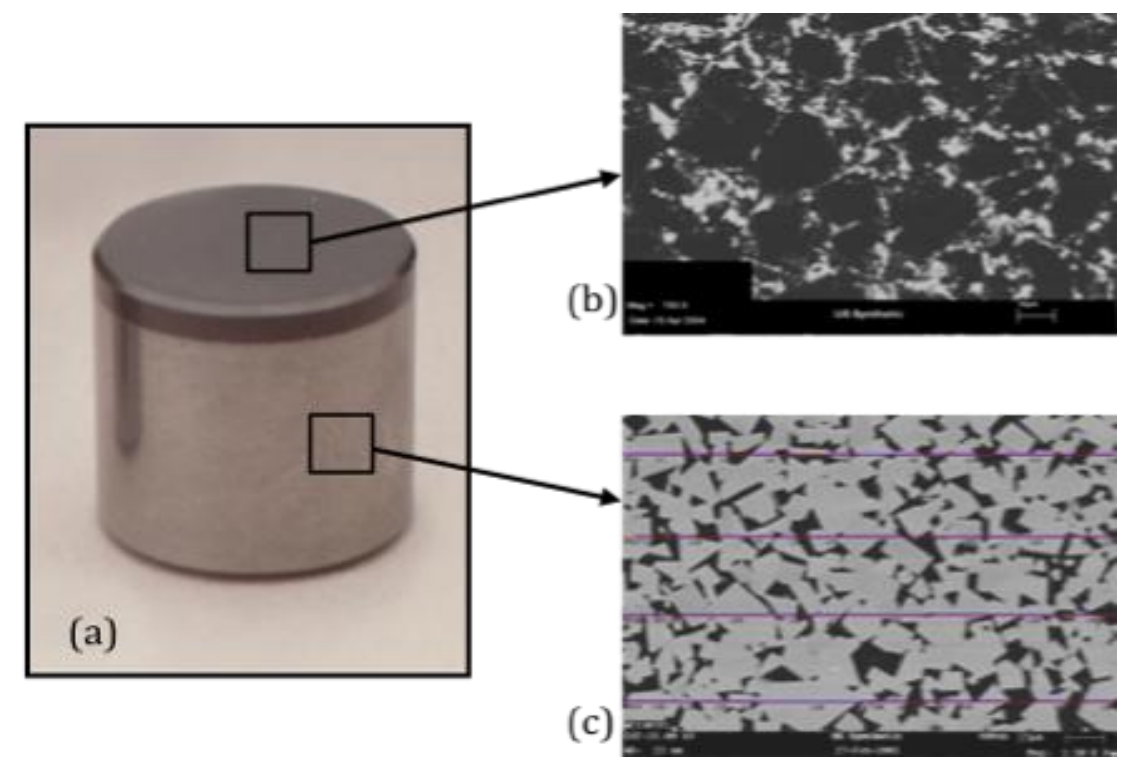

Figure 1.1: A polycrystalline diamond compact (a) with PCD layer (b) sintered onto cemented WC/Co substrate (c)

Despite these challenges, several authors have successfully measured the mechanical material properties of PCD such as Young's modulus, fracture toughness, compressive strength, tensile strength, and transverse rupture strength. Gigl [10] was the first to measure the transverse rupture strength of PCD. He machined thin, rectangular diamond slabs from PCD discs and subjected them to a three-point bending test. In another work, Lammer [2] measured the effects of grain size and cobalt content on several mechanical properties of PCD, including transverse rupture strength and fracture 
toughness. He tested transverse rupture strength by conducting three-point bending tests on PCD discs that were cut from sintered compacts and sintered as stand-alone discs. He built a statistical model of transverse rupture strength as a function of cobalt content and grain size, and concluded that transverse rupture strength is inversely related to grain size and directly related to cobalt content. Huang et al. [11] also measured the fracture toughness and transverse rupture strength of PCD. He found that the fracture toughness increases with increasing grain size, and that transverse rupture strength decreases with increasing grain size.

Others have conducted extensive tests to understand the mechanisms behind thermal degradation of PCD. Miess and Rai [3] studied the effects of grain size and residual cobalt content on the thermal-resistance of diamond layers after removing them from the substrate. They subjected diamond discs made with $5 \mu \mathrm{m}, 10 \mu \mathrm{m}$ and $30 \mu \mathrm{m}$ grain sizes to temperatures between 600 and $800^{\circ} \mathrm{C}$ in different gas environments. They observed that thermal damage begins to appear around $600^{\circ} \mathrm{C}$ in air as evidenced by intergranular microcracking. This was believed to be caused by rapid graphitization of the diamond grains, and also by residual stresses within the material that exist due to the different thermal expansion rates of diamond and cobalt. They investigated these stresses using Raman spectroscopy, which revealed that the $5 \mu \mathrm{m}$ samples had a compressive stress approximately four times higher than the coarse grain samples. They concluded that increasing amounts of the cobalt phase in PCD layers reduces thermal-resistance.

These studies show two important things; first, the transverse rupture strength and thermal-resistance of PCD are related to the amount of cobalt in the material, and second, thermal-resistance is inversely proportional to the cobalt content while transverse rupture 
strength is directly proportional to the cobalt content. This implied tradeoff between the objectives is what we seek to characterize.

However, in order to make this characterization we need to understand how to control the amount of residual cobalt in the diamond. Unlike cemented tungsten carbide, the cobalt content of PCD is not an independent variable that the designer specifies upfront. As Lammer points out, the cobalt is introduced by infiltration and not by admixing, and therefore it cannot be systematically changed for a given grain size [2]. We would like to mention here that the latter comment may be true when all other material and process variables are fixed. However, there are other design variables involved in the process that may allow a designer to alter cobalt content for a given grain size, and we will discuss those in the next chapter.

We would like to point out that although thermal-resistance is an important concept for PCD that is related to the cobalt content, it is difficult to quantify in a generally meaningful way. Unlike tensile strength, there is no standard measure for this property. For clarity, the term "thermal-resistance" as used in this paper refers to the ability of the material to resist thermal degradation, and is not in any way related to the term commonly used in heat transfer. Mehan and Hibbs [12] correlated thermal damage in PCD to the number of acoustic events emitted from PCD compacts that were rapidly heated to elevated temperatures. They validated this correlation by measuring the wear rates of the heated compacts in rock cutting tests. They report that an incubation period exists before the onset of thermal damage, and report that the onset of damage occurs sooner in fine grain PCD than in coarse grain PCD. They propose that an Arrhenius relation in terms of time and temperature can explain thermal damage in some PCD 
compacts. However, they do not draw any correlation between thermal damage and cobalt content. Therefore, because quantifying thermal-resistance is open to question at this time, we have chosen to use the residual cobalt content as the substitute objective for thermal-resistance, recognizing it has a general inverse relationship to thermal-resistance. We leave the development of an explicit relationship between thermal-resistance and cobalt content for future work.

Finally, the number of PCD manufacturers has grown substantially in recent years, especially in countries where labor costs are low. This increase in the number of PCD suppliers has caused an increase in cost-sensitivity by PCD consumers. As a result, manufacturers must become more aware of the costs they incur when developing new materials. Therefore, an understanding of how manufacturing cost is influenced by improving transverse rupture strength and thermal stability is important.

The goals of this thesis are (1) to provide designers of PCD with a useful characterization of the tradeoff between the competing material property objectives of thermal stability (i.e. cobalt content) and transverse rupture strength, and (2) to examine the influence of this tradeoff on the manufacturing costs associated with improving these objectives. The next section outlines the research approach that was used to accomplish these goals.

\subsection{Research Approach}

In order to accomplish the goals set forth in the previous section, the following research approach was used: 
(1) Empirical models were developed between the design variables of sintering pressure and grain size, and the material properties of residual cobalt content and transverse rupture strength in PCD.

(2) The tradeoff between the cobalt content and transverse rupture strength was characterized using the normal constraint method.

(3) The tradeoff between manufacturing cost and residual cobalt content was characterized using the normal constraint method.

(4) The tradeoff characterizations were used to select the optimal PCD designs.

(5) Wear rate models of PCD were developed for two different rock cutting tests using the same design variables from (1) and (2), and

(6) The optimal PCD designs were evaluated in terms of the wear rate models developed in (5).

In order to accomplish (1)-(3), we followed the iterative 3-step process illustrated in Figure 1.2. This process shows how the Pareto frontiers can be generated from empirically based objectives. Step 3 in the process involves evaluating the adequacy of the Pareto frontier. Part of the thesis was to establish a method for making this evaluation meaningful.

The balance of this thesis is presented as follows. Chapter 2 presents the development of the Pareto frontiers using the method of Figure 1.2, outlining in detail the experimental process, the test methods and measurements, and the development and refinement of the empirical models used to make the tradeoff characterizations. In Chapter 3, we use the tradeoffs developed in Chapter 2 for selecting the optimal PCD 
designs, paying special attention to the influence of the manufacturing cost. We also show the development of the wear rate models and compare the best wear rate designs to the Pareto frontier models in order to validate our assumption that characterizing these tradeoffs can help engineers design better wear tools. Finally, Chapter 4 provides conclusions and future work.

STEP 1

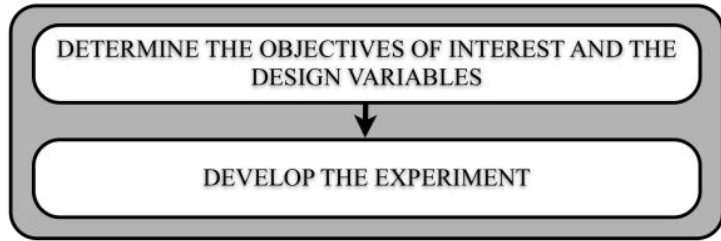

STEP 2

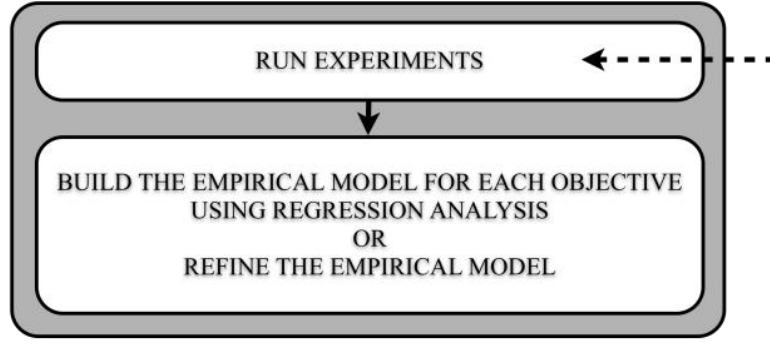

STEP 3

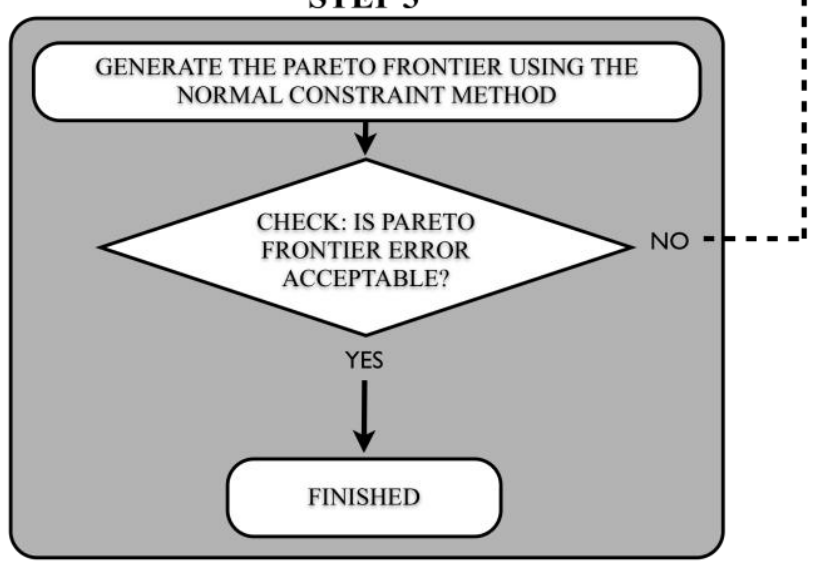

Figure 1.2: Three-step approach for developing the Pareto frontier model 


\section{Development of Pareto Frontier Models}

In this chapter, the tradeoff characterization of transverse rupture strength, cobalt content, and manufacturing cost is presented using empirical models for the first two objectives. Because the empirical models that were used to generate the Pareto frontier were approximated over a wide variable range and contain experimental error, this introduced uncertainty in the Pareto frontier. One way to decrease this uncertainty would be to build a second empirical model over a smaller range of the variables, in the areas of interest. However, this was not possible due to the high experimental cost, and may not have provided a better approximation given the small the signal-to-noise ratio in the transverse rupture strength. Therefore, a method was needed for describing the uncertainty in the Pareto frontier. This chapter describes such a method.

This chapter begins by describing the experimental program that was used to develop the empirical models for transverse rupture strength and cobalt content. This includes establishing the important design variables for PCD, the design variable range, and the test sample preparation. The test methods and measurements are described, and the empirical models for cobalt content and transverse rupture strength are presented in terms of sintering pressure and grain size. Then the manufacturing cost objective and the design variables that influence it are discussed. This brings us through Step 2 of the process illustrated in Figure 1.2. 
Last, the development of the Pareto frontier is presented using the normal constraint method, and a process for characterizing the uncertainty in a Pareto frontier generated from empirically-based objectives is illustrated.

Throughout the remainder of this thesis we will refer to the objectives of cobalt content, transverse rupture strength, and manufacturing cost as $\mu_{1}, \mu_{2}$, and $\mu_{3}$ respectively.

\subsection{Experimental Program}

Response Surface Methodology is a useful experimental approach for exploring the relationship between a group of design variables and one or more response variables. This method was first introduced by Box and Wilson in 1951 [13] and has found wide application in the design, development and optimization of new and existing processes and products [14]. This approach is also useful when experiments are costly because it requires a relatively small number of experiments in order to generate an empirical relationship between the design variables and the response. We now discuss the application of this method to the development of empirical models for $\mu_{1}$ and $\mu_{2}$.

The first step in Response Surface Methodology is to determine the important design variables that have an effect on the response. From the literature, the initial diamond grain size is under the engineer's control and has a significant effect on transverse rupture strength and cobalt content [2-3,10-11]. Other variables known to have an important effect on diamond sintering are the process variables of pressure, time and temperature [15]. For this study, we chose to include sintering pressure $\left(x_{1}\right)$ and diamond grain size $\left(x_{2}\right)$ as the factors of interest and maintained a constant press time and temperature. The main reasons behind this are related to the constraints imposed on time 
and temperature from a business perspective (i.e. extremely long sintering times limit capacity, and high temperatures have a negative effect on tool life).

The second step is to select a model that will best describe the relationship between the design variables and the response. This is difficult to do when we do not know what the relationship looks like between the objectives and the design variables. The best we can do is to try to draw some reasonable conclusions about the form of the relationships from the work of others who have studied one design variable at a time $[2,11,16]$. Based on previous work, the relationship between transverse rupture strength and grain size is likely linear [11], but may be approximated by a second-order polynomial [2]. Other work indicates that the relationship between cobalt content and grain size is best approximated by a second-order polynomial, and that cobalt content and pressure are linearly related [16]. With this information, we chose the second-order polynomial model proposed by Box and Wilson [13]. This model is widely used in industrial applications because it can be adapted to situations where the relationship between the response and the design variables is both linear and quadratic. It can also model any interaction between the design variables that might exist. The general mathematical representation of this model for two factors is

$$
\mu=\beta_{0}+\beta_{1} x_{1}+\beta_{2} x_{2}+\beta_{3} x_{1}^{2}+\beta_{4} x_{2}^{2}+\beta_{5} x_{1} x_{2}
$$

The $\beta$ coefficients are estimated from the experimental data using least squares regression.

For the experiment design, we chose a central composite design with two factors [17]. This design is advantageous because it requires only nine experimental runs in order to estimate the coefficients. The first four runs test all combinations of the high and 
low values of the design variables. The fifth run is called the "center point" because it tests the middle values of each design variable. The last four runs are called "star points" and are denoted by $\alpha$. These runs allow for the estimation of curvature within each factor and more fully cover the experimental region.

The third step is to determine the experimental values of the design variables. The lower limit of pressure is fixed by the intersection of the diamond-graphite transition line, shown in Figure 2.1, with the melting point of the cobalt alloy $\left(\sim 1400^{\circ} \mathrm{C}\right)$, illustrated by the left edge of the red triangle in Figure 2.1. This intersection gives a lower pressure limit of approximately 55 kbar [18]. The upper limit is constrained by the safe operating range of the press. Grain sizes were selected based on the common extremes of $12 \mu \mathrm{m}$ and $70 \mu \mathrm{m}$. With the extreme levels fixed for each variable, the center value was taken as the midpoint between the extremes, and the low/high values were calculated in relation to the coded values. The design variable levels are listed in Table 2.1.

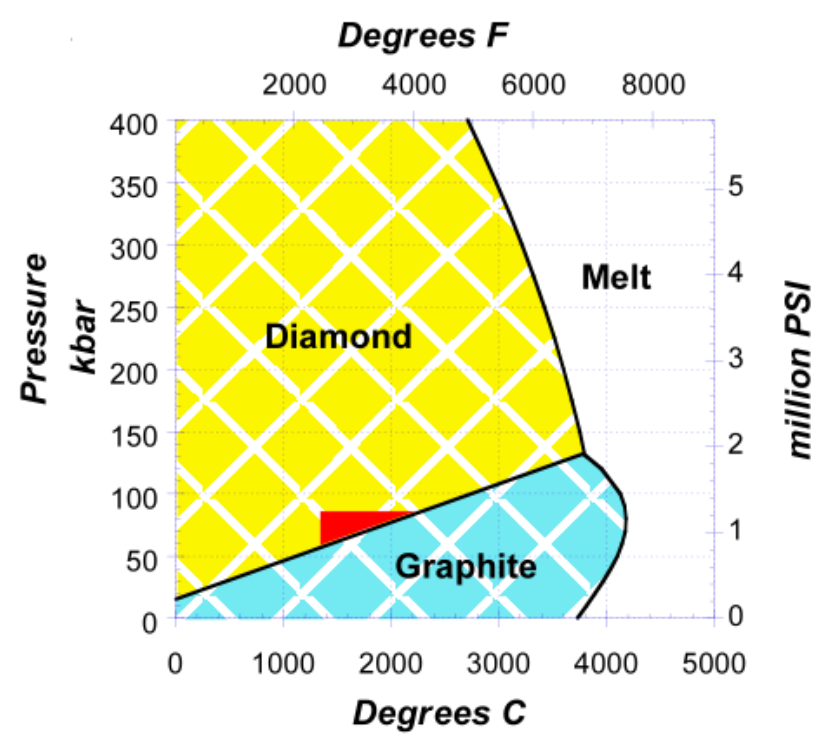

Figure 2.1: Pressure-Temperature phase diagram for carbon 
Table 2.1: Design variable values in coded (parenthesis) and uncoded form

\begin{tabular}{cccccc}
\hline Low $\boldsymbol{\alpha}$ & Low & Center & High & High $\boldsymbol{\alpha}$ \\
$(\mathbf{( - \sqrt { } )}$ & $(-\mathbf{1})$ & $(\mathbf{0})$ & $\begin{array}{c}(\mathbf{1}) \\
(\sqrt{\mathbf{2}})\end{array}$ \\
\hline Pressure $(\mathrm{kbar})$ & 55 & 58.2 & 66 & 73.8 & 77 \\
Grain Size $(\mu \mathrm{m})$ & 12 & 20 & 40 & 60 & 70 \\
\hline
\end{tabular}

The last step is to generate the experimental run table and build the samples accordingly. The experimental runs are illustrated in the design variable space shown in Figure 2.2. All PCD samples were sintered on WC/Co substrates using a cubic press apparatus, finished to a diameter of $15.875 \mathrm{~mm}( \pm 0.025 \mathrm{~mm})$ and then subsequently removed from the substrate by wire EDM. The PCD samples were then lapped to a thickness of $1.016 \mathrm{~mm}( \pm 0.025 \mathrm{~mm})$ using 300 mesh diamond grit.

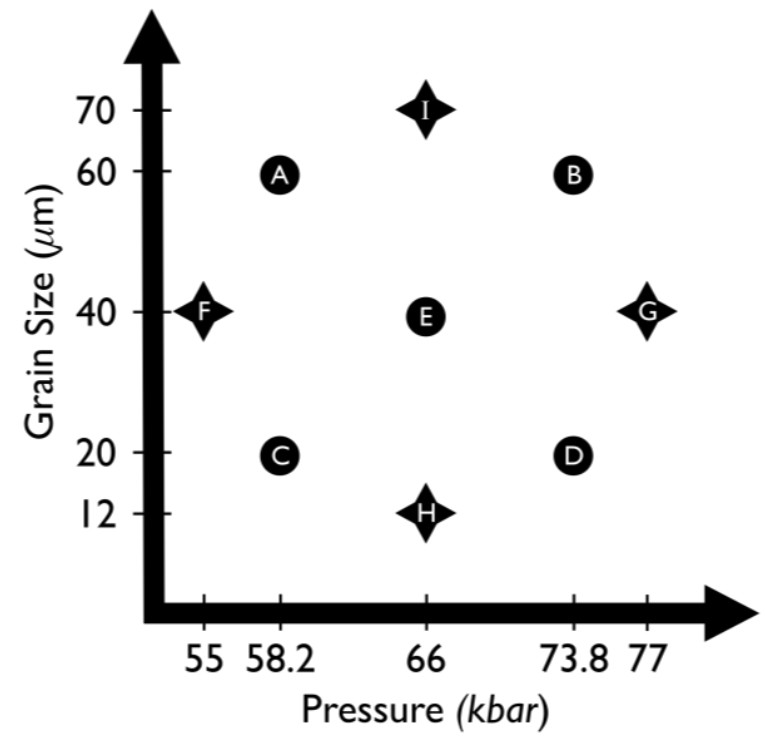

Figure 2.2: Experimental runs shown in the design variable space 


\subsection{Test Methods and Measurements}

After finishing the samples to the specified geometry, we took measurements of the thickness, mass and cobalt content of each sample. The cobalt content was calculated by measuring the magnetic moment-to-weight ratio of a PCD sample, calculating the weight-specific saturation magnetization of that sample, and then using the magnetic material constant for Co-WC alloy to find the weight-percent of the cobalt in each sample. These measurements are summarized in Table 2.2.

Table 2.2: Summary statistics for experimental runs A - I (10 samples each)

\begin{tabular}{cccccccccc}
\hline Measurement & A & B & C & D & E & F & G & H & I \\
\hline $\begin{array}{c}\text { Mean Disc } \\
\text { Thickness (mm) }\end{array}$ & 1.035 & 1.027 & 1.029 & 1.032 & 1.035 & 1.017 & 1.037 & 1.039 & 1.035 \\
\hline $\begin{array}{c}\text { Standard } \\
\text { Deviation (mm) }\end{array}$ & 0.007 & 0.007 & 0.009 & 0.012 & 0.010 & 0.014 & 0.008 & 0.005 & 0.011 \\
\hline $\begin{array}{c}\text { Mean Disc } \\
\text { Mass (g) }\end{array}$ & 0.793 & 0.768 & 0.817 & 0.788 & 0.785 & 0.793 & 0.782 & 0.821 & 0.776 \\
\hline $\begin{array}{c}\text { Standard } \\
\text { Deviation (g) }\end{array}$ & 0.005 & 0.007 & 0.006 & 0.009 & 0.007 & 0.013 & 0.005 & 0.003 & 0.006 \\
\hline $\begin{array}{c}\text { Mean Cobalt } \\
\text { Content } \\
\text { (Wt.\%) }\end{array}$ & 6.595 & 4.926 & 8.019 & 5.873 & 5.723 & 7.359 & 5.283 & 7.802 & 5.299 \\
\hline $\begin{array}{c}\text { Standard } \\
\text { Deviation } \\
\text { (Wt.\%) }\end{array}$ & 0.087 & 0.052 & 0.158 & 0.098 & 0.277 & 0.126 & 0.089 & 0.123 & 0.058 \\
\hline
\end{tabular}

Once the measurements were obtained, each PCD sample was subjected to a transverse rupture strength test. Figure 2.3 shows a simple schematic of the test. While supported with an aluminum barrel at the diameter on one face, the opposite face of the disc was pressurized by hydraulic fluid until failure. The pressure at failure was recorded 
and the rupture stress $\left(\sigma_{\mathrm{R}}\right)$ calculated using the relation [19]

$$
\sigma_{R}=\frac{3 \operatorname{Pr}^{2}(3+v)}{8 t^{2}}
$$

A summary of the transverse rupture strength measurements is given in Table 2.3. The mean values are within the range of previous measurements $[2,10,11]$, and the standard deviation in designs $\mathrm{E}-\mathrm{I}$ is within the range reported by Lammer [2]. The standard deviation in the measurements for designs $A-D$ is significantly lower compared to the other designs. An inspection of the samples and the testing system did not reveal any cause for this inconsistency, and therefore was assumed to be coincidental.
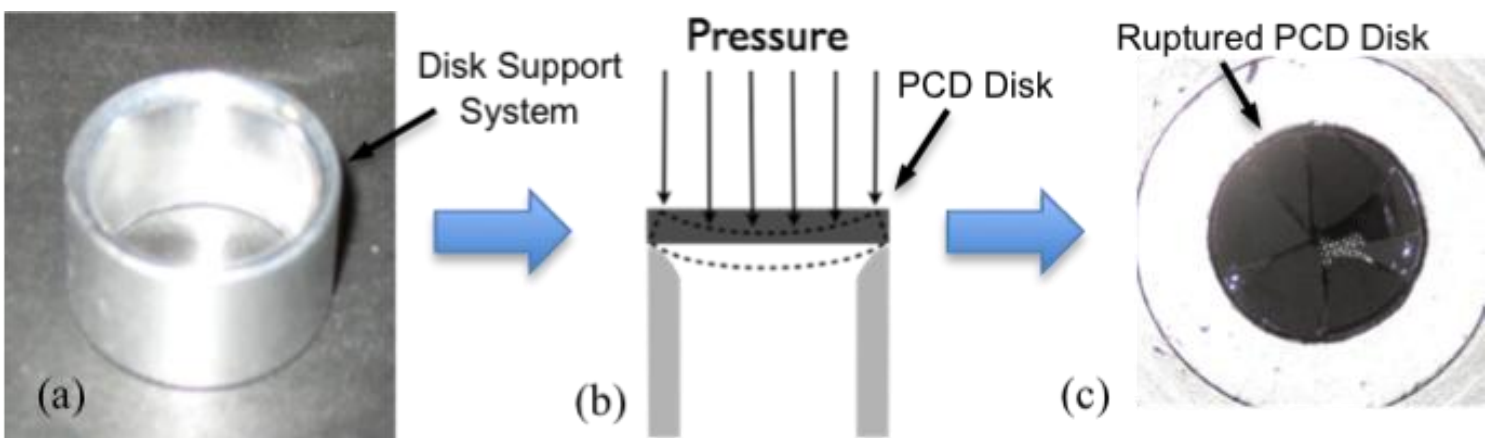

Figure 2.3: Transverse rupture strength test setup

Table 2.3: Summary of transverse rupture strength measurements

\begin{tabular}{cccccccccc}
\hline Measurement & $\mathbf{A}$ & $\mathbf{B}$ & $\mathbf{C}$ & $\mathbf{D}$ & $\mathbf{E}$ & $\mathbf{F}$ & $\mathbf{G}$ & $\mathbf{H}$ & $\mathbf{I}$ \\
\hline \hline $\begin{array}{c}\text { Mean } \sigma_{\mathrm{R}} \\
(\mathbf{M P a})\end{array}$ & 1043 & 862 & 1435 & 1349 & 838 & 1169 & 980 & 1184 & 592 \\
\hline $\begin{array}{c}\text { Standard } \\
\text { Deviation } \\
(\mathbf{M P a})\end{array}$ & 64.83 & 42.42 & 55.67 & 85.63 & 221.6 & 246.6 & 154.3 & 261.5 & 178.2 \\
\hline
\end{tabular}




\subsection{Response Surface Model for Cobalt Content}

The coefficients in equation (2.1) are estimated from the data using least squares regression. Analysis of the cobalt content data gives the relationship

$$
\mu_{1}=5.723-.844 x_{1}-.739 x_{2}+.278 x_{1}^{2}+.393_{4} x_{2}^{2}+.119 x_{1} x_{2}
$$

where $\mu_{1}$ is in percent-by-weight and the variables $x_{1}$ and $x_{2}$ are in coded form. Figure 2.4 shows a graphical representation of this model. Recall from the previous discussion that thermal-resistance is inversely related to cobalt content, therefore lower values of cobalt are more desirable for this objective.

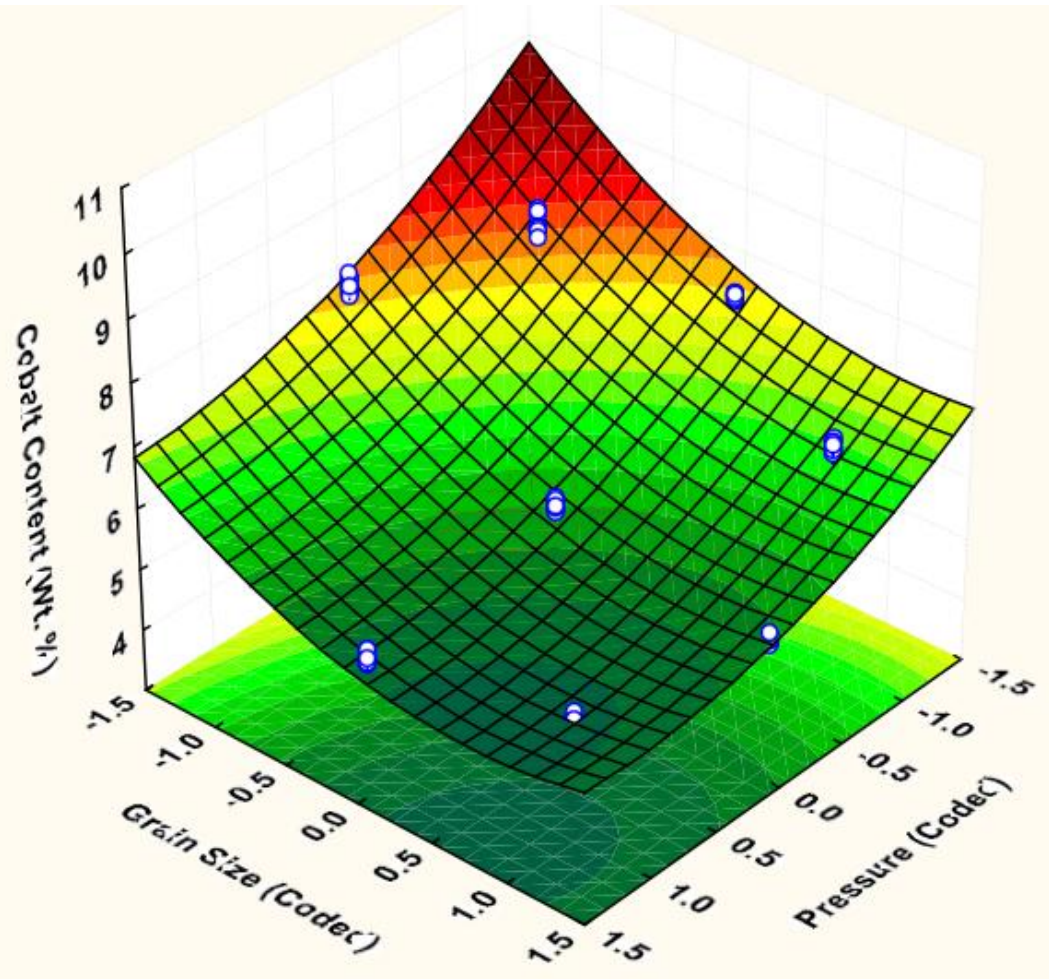

Figure 2.4: Cobalt content response surface model plotted against the experimental data

A few important observations can be made from this model. First, the cobalt content is consistent with the trends in the literature for grain size (i.e. cobalt content 
increases with decreasing grain size [2-3]). Second, it was previously reported that cobalt content cannot be changed systematically for a given diamond grain size [2]. However, we can clearly see that the cobalt content for a fixed grain size can be changed by adjusting the pressure. This is important because it gives the designer another degree of freedom for controlling the cobalt content objective.

\subsection{Response Surface Model for Transverse Rupture Strength}

The regression analysis of the transverse rupture strength data gives the relationship

$$
\mu_{2}=838.7-66.78 x_{1}-214.4 x_{2}+166.0 x_{1}^{2}+72.48_{4} x_{2}^{2}-23.92 x_{1} x_{2}
$$

where $\mu_{2}$ is in MPa and the variables $x_{1}$ and $x_{2}$ are in coded form. Figure 2.5 illustrates the response surface for this model.

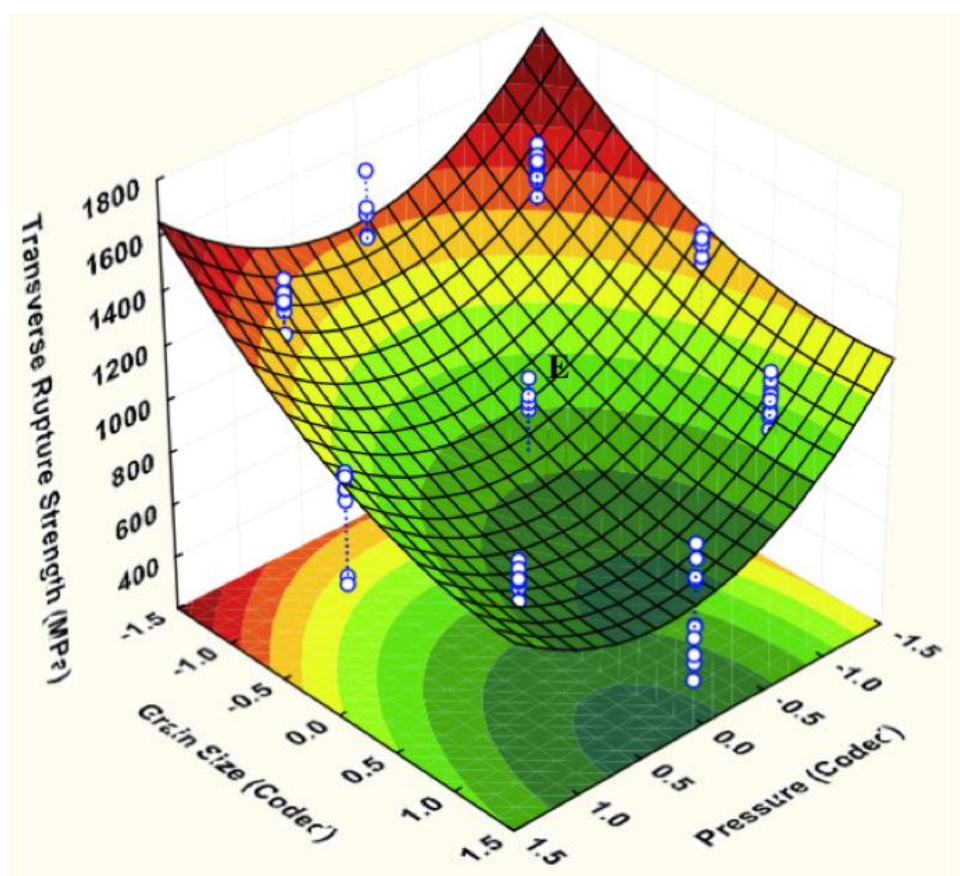

Figure 2.5: Transverse rupture strength response surface model 
The first thing we notice in Figure 2.5 is that the transverse rupture strength increases with decreasing grain size, which is consistent with the trend reported by Lammer [2]. We also notice a large amount of scatter in this data compared to the cobalt content data. This type of scatter is expected when subjecting brittle materials to bending stresses because of their sensitivity to flaws in the samples.

Next, notice that for a constant grain size the model predicts transverse rupture strength to be lowest at a pressure of $66 \mathrm{kbar}$. This curvature trend in the model might be an artifact of the different variances among individual data sets, and will be validated by running additional experiments and comparing those results to the predictions of the model. The results of these additional experiments are shown in the next section. Finding the correct empirical model between the design variables and the response is important because the Pareto frontier is generated using the empirical models. An alternative model to the quadratic may be needed if there exists a large discrepancy between the Pareto frontier model and the actual Pareto frontier that we observe in the data. This is why we have added the step in the Pareto frontier development process to check for acceptable error in the Pareto frontier model (see Figure 1.2).

\subsection{Manufacturing Cost Model}

In Chapter 1 we mentioned that the equipment for manufacturing PCD is costly to maintain. This is largely due to the high replacement costs associated with the tungsten carbide anvils used in a typical high pressure, high temperature diamond press. Because the anvils experience dozens of cycles per day, any increase in stress due to an increase in pressure and/or temperature will reduce the tungsten carbide fatigue life. No general model was found in the literature that describes cost as a function of the design variables. 
However, for this paper we obtained a model from an experienced manufacturer of PCD that shows the percent increase in manufacturing cost as a function of pressure. The model is given as

$$
\mu_{3}=.04058 e^{1.0595 x_{1}}-.00912
$$

where $\mu_{3}$ is the percent increase in cost and $x_{1}$ is the pressure in coded units. Note that $\mu_{3}$ is equal to zero at the lowest possible sintering pressure (55 kbar, or - 1.41 in coded units). This model assumes that diamond grain size has no affect on the increase in cost.

To this point in the thesis we have completed the first two steps of the Pareto frontier development process outlined in Figure 1.2. We determined the important design variables, and developed and executed the experimental program. We then developed empirical models that describe cobalt content and transverse rupture strength as functions of sintering pressure and diamond grain size. These models are useful because they show the designer how to manipulate the design variables to meet a desired value of the objective.

Another potential use for these models relates to benchmarking products made by different manufacturers. If the designer could measure cobalt content in a product manufactured at unknown conditions of pressure and grain size, he could use the response surface to find the most likely values of these design variables that would produce that particular product. And if one of the design variables were known, by calculation or test or inspection, the other could be estimated from the empirical relation. This could potentially provide competitive benchmarking information to engineers and managers involved in new product development. 
With empirical relationships established between the design variables of interest and the objectives, we can now proceed to Step 3 in the Pareto frontier development process. Before doing so however, we will briefly review the development of the normal constraint method and its advantages in characterizing tradeoffs between competing objectives.

\subsection{The Normal Constraint Method}

The normal constraint method was developed by Messac and Mattson [20] and is an effective way to find the Pareto frontier between any number of competing objectives. The method is especially useful when large differences in scale exist between design objectives because it can be normalized to provide an even distribution of Pareto solutions [18]. This set of solutions is guaranteed not to over represent or under represent any single area of the Pareto frontier, which ensures that the designer does not miss out on potentially optimal designs [17].

In this section we present the normal constraint method for the bi-objective case, where both objectives are to be minimized, and refer the reader to Messac and Mattson [5] for the development of the general $n$-dimensional case. Note that a maximization problem can be turned into a minimization problem by changing the sign of the objective. A graphical representation of this method is shown in Figure 2.6. 


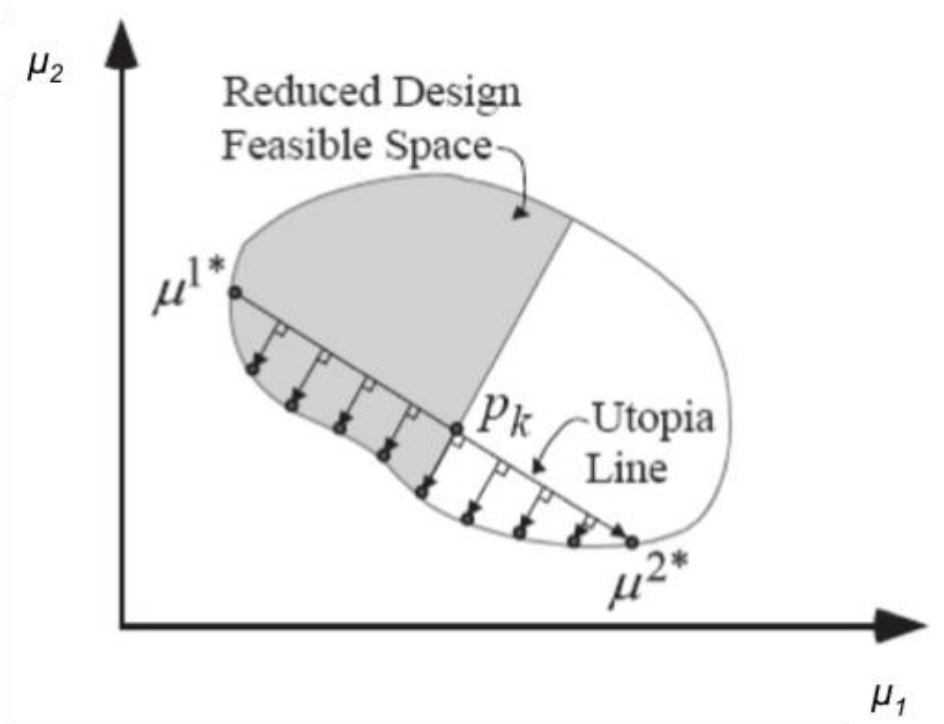

Figure 2.6: Graphical representation of the normal constraint method for bi-objective problems [17]

The process [21] of generating the Pareto frontier is as follows.

Step 1 -Obtain Anchor Points: Obtain the ith anchor point $\mu^{1^{*}}$ and $\mu^{2^{*}}$, by

$$
\begin{aligned}
& \mu_{x}^{i^{*}}=\min \mu_{i} \\
& \text { s.t. } \\
& g(x) \leq 0 \\
& h(x)=0 \\
& x_{L} \leq x \leq x_{U}
\end{aligned}
$$

Step 2 -Define utopia line vector: In this case, the utopia line vector is

$$
\vec{v}=\mu^{1^{*}}-\mu^{2^{*}}
$$

Step 3 -Compute increments: Compute an increment $\delta$ along the vector $\vec{v}$ for a prescribed number of solutions, $m$, along the vector as 


$$
\delta=\frac{1}{m-1}
$$

Step 4-Generate utopia line points: Evaluate the $k$ th point on the utopia line as

$$
p_{k}=\alpha_{k} \mu^{*}
$$

where the non-dimensional parameter $\alpha_{k}$ satisfies the conditions

$$
0 \leq \alpha_{k} \leq 1
$$

and

$$
\sum_{k=1}^{m} \alpha_{k}=1
$$

By varying $\alpha_{k}$ from 0 to 1 with a fixed increment of $\delta$, a distribution of points on the utopia line can be generated.

Step 5 - Generate Pareto points: Solve Problem 1 for a set of Pareto solutions for each point $p_{k}$ generated in Step 4. Do this for the prescribed number of solutions $m$.

Problem 1: Normal constraint bi-objective optimization problem for point $p_{k}$

$$
\min _{x} \mu_{2}(x)
$$

subject to

$$
\begin{gathered}
g(x) \leq 0 \\
h(x)=0 \\
x_{l} \leq x \leq x_{u}
\end{gathered}
$$

where $g(x)$ includes the inequality constraint

$$
\vec{v} \cdot\left(\mu-p_{k}\right) \leq 0
$$


The normal constraint method can be implemented with any optimization software package. Equations (2.6) through (2.15) were used to generate the Pareto frontier by utilizing the fmincon algorithm provided in the Matlab ${ }^{\circledR}$ optimization toolbox. Next, the tradeoff model development between $\mu_{1}$ and $\mu_{2}$, and between $\mu_{1}$ and $\mu_{3}$ is presented.

\subsection{Characterizing the Tradeoff Between $\mu_{1}$ and $\mu_{2}$}

\subsubsection{Iteration 1}

Using equations (2.3) and (2.4) in the normal constraint method, we obtain the initial Pareto frontier model shown in Figure 2.7. The Pareto frontier represents the tradeoff between $\mu_{1}$ and $\mu_{2}$, and shows that $\mu_{2}$ increases as $\mu_{1}$ increases. This trend is what we expected to see since our goal is to minimize $\mu_{1}$ and maximize $\mu_{2}$. What we did not expect to see, however, is the existence of two distinct segments of the Pareto frontier. In

general, Segment 1 contains low values of $\mu_{1}$ at the expense of $\mu_{2}$, and Segment 2 contains high values of $\mu_{2}$ at the expense of $\mu_{1}$. The gap between each segment is a nonconvex region where no Pareto optimal solutions exist. This means that any design inbetween these two segments is dominated by a design on Segment 1 .

This brings us to the last part of Step 3 in the tradeoff development process, which is to check the adequacy of the Pareto frontier model. In order to check this, we must determine how far away from the model we can reasonably expect a true Pareto optimal design to exist. Since the Pareto frontier is generated using empirical relations that are subject to error due to variation in the experimental data, the Pareto frontier itself is also 
subject to the same error. We can quantify this error by including confidence intervals on every design point that is generated by solving Problem 1 in Section 2.6 for each point $p_{k}$.

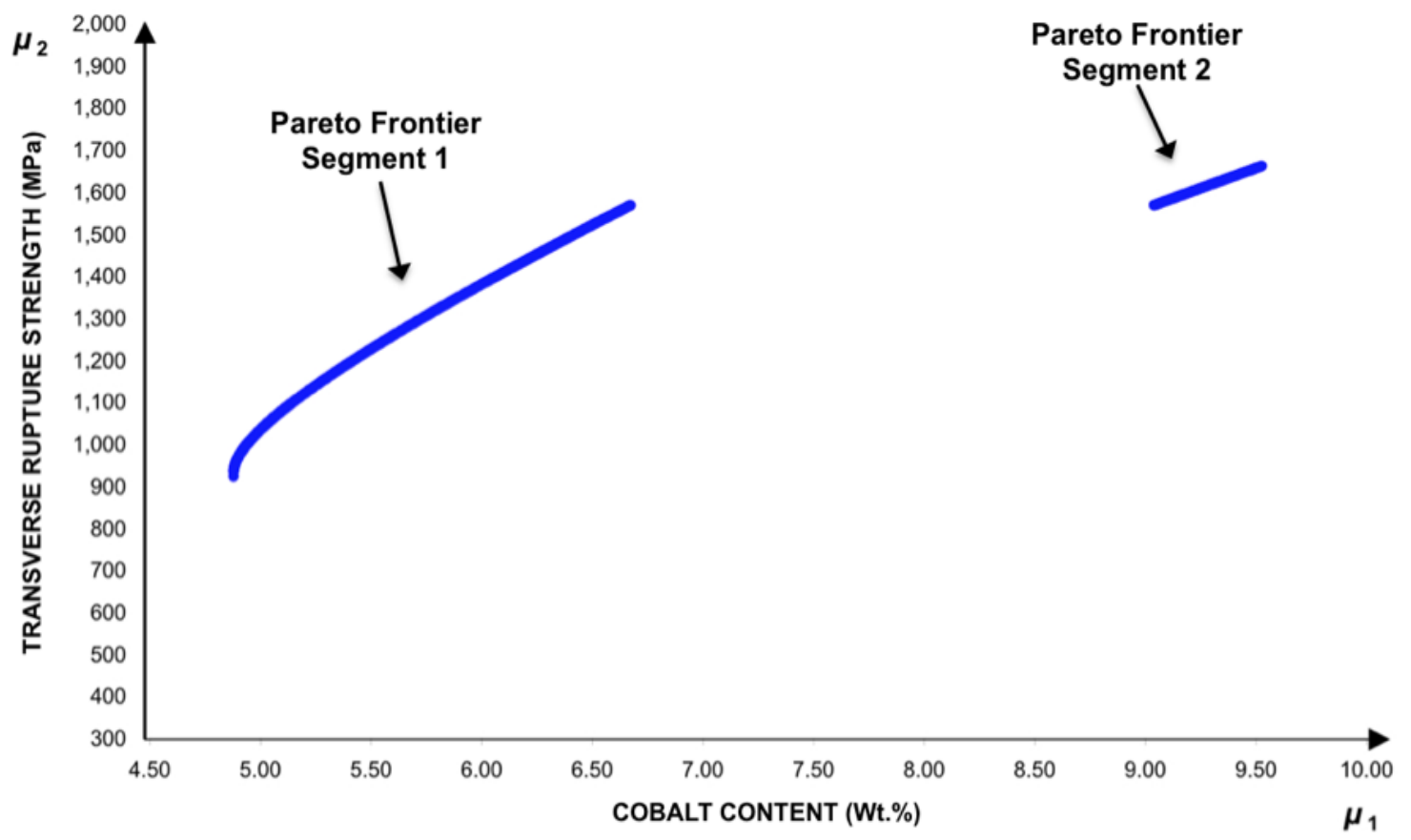

Figure 2.7: The Pareto frontier for $\mu_{1}$ and $\mu_{2}$, generated using equations (2.3) and (2.4)

For ease of illustration, we draw a region around the Pareto frontier based on the $95 \%$ confidence intervals for the mean as shown in Figure 2.8. An "adequate" Pareto frontier should capture the Pareto optimal designs from the experimental data within this region. The range of a confidence interval on a mean depends on the values of the design variables, and performing the calculations by hand can be quite rigorous [14]. We used a commercially available statistical analysis software package [22] to perform the calculation of all confidence intervals and to find the regression coefficients for the response surface equations. 


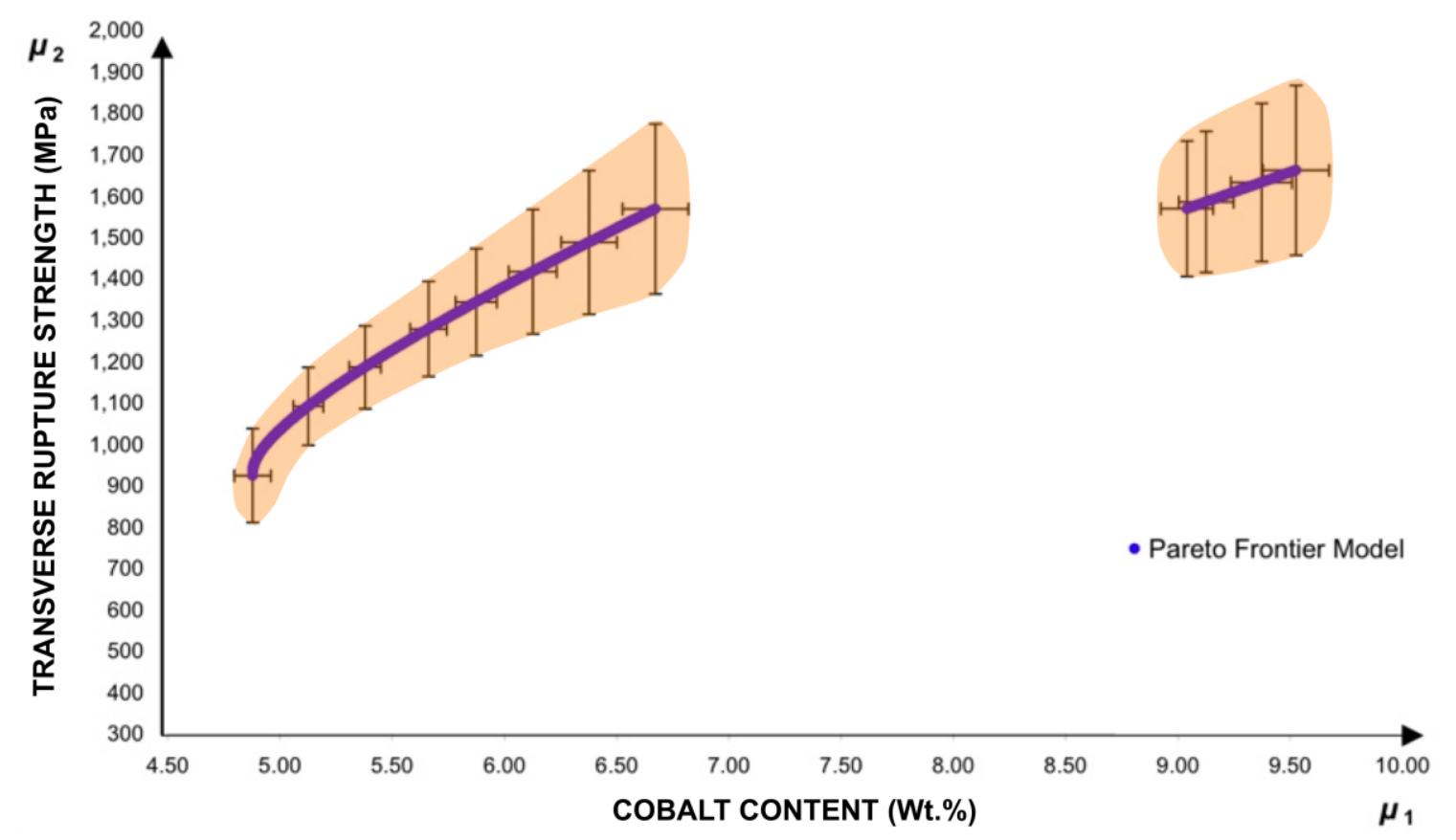

Figure 2.8: The Pareto frontier with a "confidence region" drawn around the $95 \%$ confidence limits on the mean for each segment

Figure 2.9 shows the cobalt content and transverse rupture strength data from Tables 2.2 and 2.3 plotted against the Pareto frontier model. According to the model, designs B and D can be considered Pareto optimal because they fall within the confidence region of the Pareto frontier. However, when compared to the experimental data, the Pareto optimal designs include B, C, D, and G. These are Pareto optimal because they dominate at least one of the other five experimental designs.

The Pareto frontier model only represents two out of four possible Pareto optimal designs. We consider this an inadequate representation of the data. Also, the gap in the model is questionable because we find no scientific basis for such a deficiency of Pareto optimal solutions in the range $6.60 \% \leq \mu_{1} \leq 9.00 \%$. This conclusion induces an iteration and leads us back to Step 2 in our tradeoff model development process. 


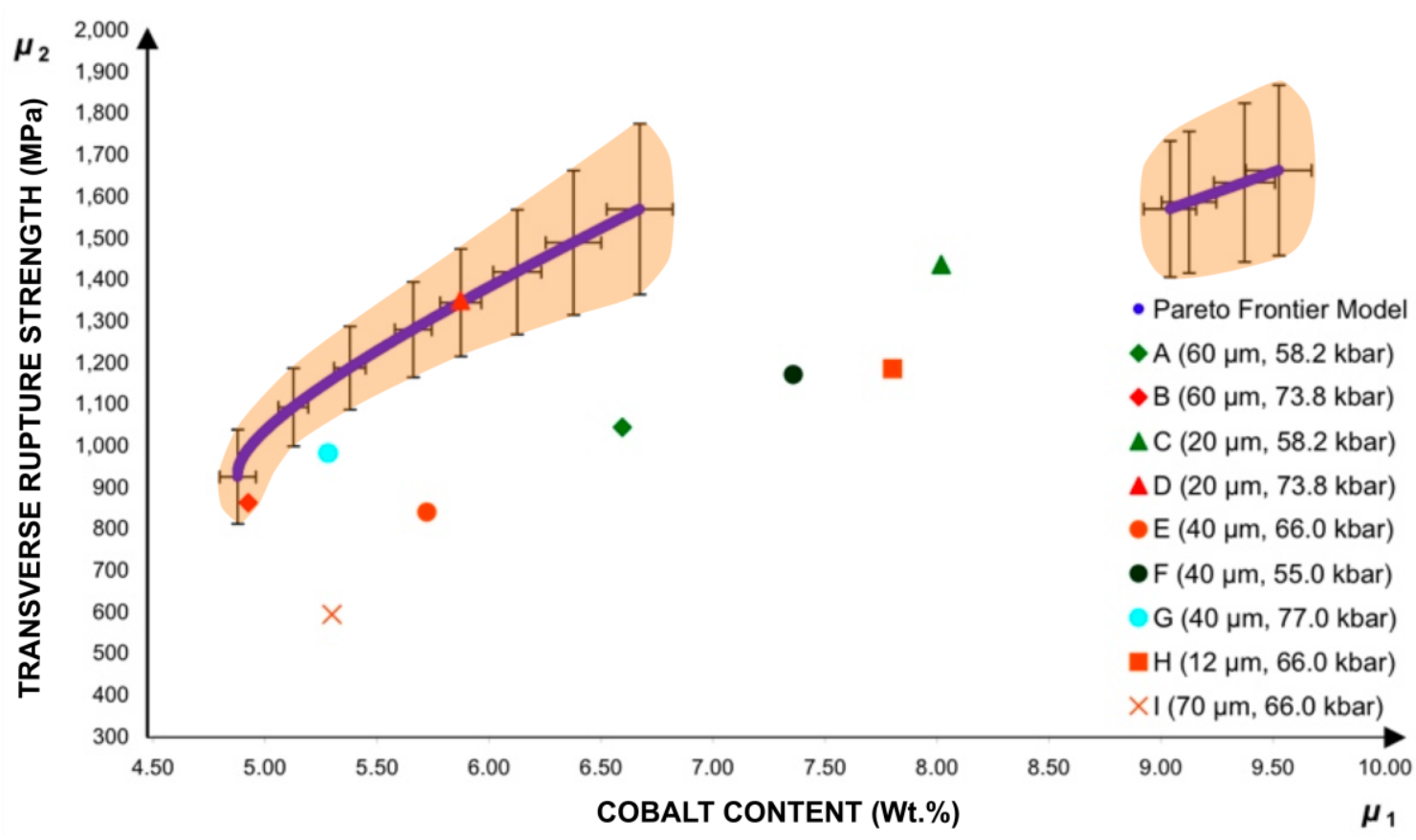

Figure 2.9: A comparison of the first Pareto frontier model to the experimental data

\subsubsection{Iteration 2}

In this section we develop a second approximation of the tradeoff relationship between $\mu_{1}$ and $\mu_{2}$ by altering equation (2.4). Although the general trend of the first Pareto frontier model is what we expected to see, we believe that the model is not a good approximation of the true Pareto frontier because (1) the model predicts that no Pareto optimal solutions exist between the range $6.60 \% \leq \mu_{1} \leq 9.00 \%$, for which we find no scientific basis, and (2) the model only captures two out of the four non-dominated data points within the confidence region, which is not an acceptable fit to the data.

In order to more fully establish confidence in the adequacy of a second Pareto frontier model, we needed to collect more data. However, since the first nine experiments were quite costly ( $\$ 33,000$ US), we could only run four more designs in two regions of interest. Since we suspect that the empirical model for transverse rupture 
strength underestimates the response at pressures of $66 \mathrm{kbar}$, we chose to run two additional experiments at 66 kbar. These are designs $\mathrm{J}$ and $\mathrm{K}$ shown in Figure 2.10. The other two designs, $\mathrm{L}$ and $\mathrm{M}$, were run at $77 \mathrm{kbar}$ using the same diamond grain sizes as $\mathrm{J}$ and $\mathrm{K}$ and are also shown in Figure 2.10. According to the first Pareto frontier model, designs $\mathrm{L}$ and $\mathrm{M}$ correspond to Pareto optimal designs along Segment 1 of the tradeoff curve. The four additional designs are summarized in Table 2.4 along with the experimental values obtained for $\mu_{1}$ and $\mu_{2}$.

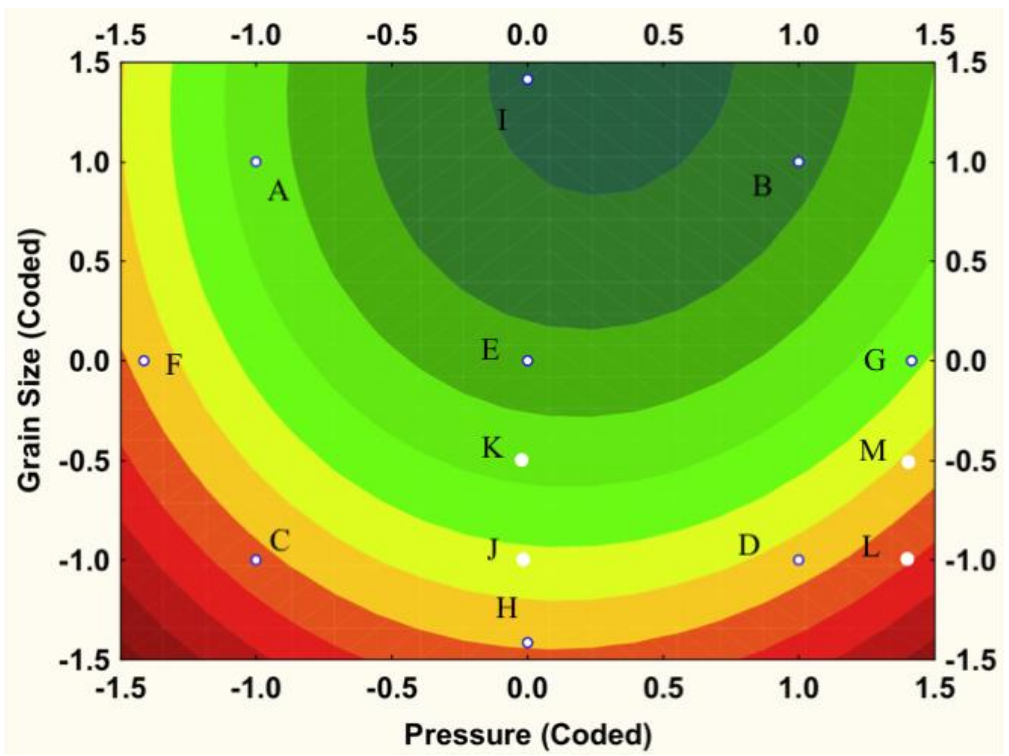

Figure 2.10: Contour plot of transverse rupture strength showing the pressure and grain size values of experiments $\mathbf{J}-\mathbf{M}$

Table 2.4: Summary of the additional experimental runs, J-M

\begin{tabular}{ccccc}
\hline & J & K & L & M \\
\hline Pressure (kbar) & 66 & 66 & 77 & 77 \\
\hline Grain Size $(\boldsymbol{\mu m})$ & 20 & 30 & 20 & 30 \\
\hline $\begin{array}{c}\text { Mean Cobalt } \\
\text { Content (Wt.\%) }\end{array}$ & 6.971 & 6.740 & 5.747 & 5.425 \\
\hline Stan. Dev. (Wt.\%) & 0.091 & 0.162 & 0.130 & 0.079 \\
\hline Mean $\sigma_{\mathrm{R}}(\mathbf{M P a})$ & 1375 & 1357 & 1204 & 1114 \\
\hline Stan. Dev. $(\mathbf{M P a})$ & 214.1 & 193.5 & 214.4 & 197.4 \\
\hline
\end{tabular}


With this additional data, we were able to examine the accuracy of equation 2.4 in predicting values of $\mu_{2}$. Figure 2.11 is a plot of the average values for transverse rupture strength for designs $\mathrm{C}, \mathrm{J}$, and $\mathrm{D}$ compared to the average values predicted by equation 2.4 (Quadratic Model) with 95\% confidence intervals. From this graph we notice that (1) the trend in the average values is fairly linear and not quadratic as equation 2.4 predicts, and (2) the predictions for designs $\mathrm{C}$ and $\mathrm{D}$ are close to falling within the confidence intervals, but the prediction for design $\mathrm{J}$ is significantly under-estimated.

The second Pareto frontier model was developed by eliminating the statistically insignificant terms from the response surface model of equation (2.4) - the $x_{1} x_{2}$ and $x_{2}^{2}$ terms - and then eliminating the $x_{1}^{2}$ term. The judgment for statistical significance is based on the $p$-value for each $\beta$ term in the regression model. We chose to drop the $x_{1} x_{2}$ and $x_{2}{ }^{2}$ terms because they have higher $p$-values compared to the rest of the $\beta$ coefficients as shown in Table 2.5. The $p$-value represents the probability that the $\beta$ coefficient is zero. Since the chances are high that the $\beta$ coefficients are zero for these, we will remove them from the model. The basis for eliminating the third term is that the scatter in the data is causing the regression estimate of curvature to be inflated as illustrated by Figure 2.11 . 


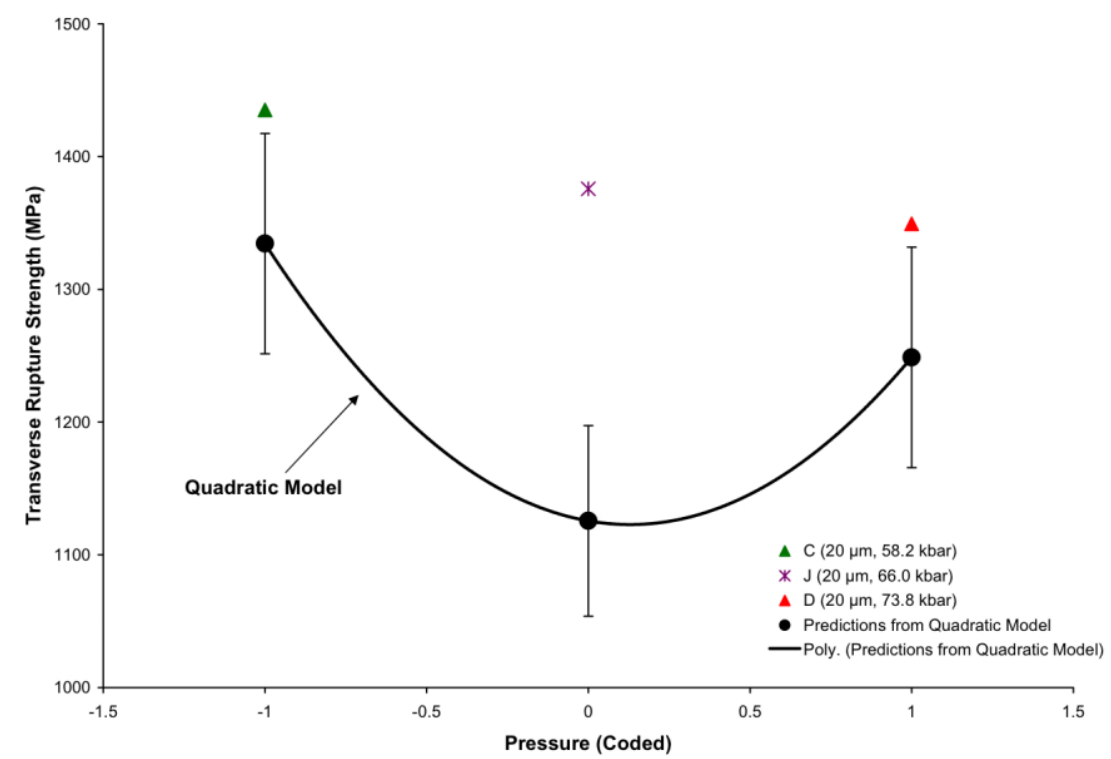

Figure 2.11: Average values compared to quadratic model predictions of $\mu_{2}$ for designs $\mathrm{C}, \mathrm{D}$ and $\mathrm{J}$

Table 2.5: Output data from Statistica used to obtain equation (2.4)

\begin{tabular}{c|c|c|c|c|c|c|c|}
\cline { 2 - 8 } Factor & Effect & $\begin{array}{c}\text { Std.Err. } \\
\text { Pure Err }\end{array}$ & $\mathrm{p}$ & $\begin{array}{c}-95 \% \\
\text { Cnf.Limt }\end{array}$ & $\begin{array}{c}+95 \% \\
\text { Cnf.Limt }\end{array}$ & Coeff. & $\begin{array}{c}\text { Std.Err. } \\
\text { Coeff. }\end{array}$ \\
\hline Mean/Interc. & 838.7 & 52.75 & 0.000000 & 733.7 & 943.7 & 838.7 & 52.75 \\
\hline$x_{1}$ & -133.6 & 37.30 & 0.000582 & -207.8 & -59.35 & -66.78 & 18.65 \\
\hline$x_{1}{ }^{2}$ & 332.0 & 61.85 & 0.000001 & 208.9 & 455.0 & 166.0 & 30.93 \\
\hline$x_{2}$ & -428.8 & 37.30 & 0.000000 & -503.0 & -354.6 & -214.4 & 18.65 \\
\hline$x_{2}^{2}$ & 144.9 & 61.85 & 0.021557 & 21.88 & 268.0 & 72.45 & 30.93 \\
\hline$x_{1} x_{2}$ & -47.8 & 52.75 & 0.367214 & -152.8 & 57.12 & -23.9 & 26.38 \\
\hline
\end{tabular}

Removing these terms and re-analyzing the data gives a linear model for $\mu_{2}$, where

$$
\mu_{2}=1050.7-66.78 x_{1}-214.4 x_{2} .
$$

This model shows that $\mu_{2}$ decreases with increasing pressure and grain size. Figure 2.12 compares the average values of $\mu_{2}$ for designs $\mathrm{C}, \mathrm{D}$ and $\mathrm{J}$ with their predictions from equation (2.4) and equation (2.16), which are listed as the quadratic model and linear model, respectively. Although the new linear model still under-predicts the actual 
average values, the discrepancy between the prediction for design $\mathrm{J}$ and the actual value is much smaller. The reason that both models under-predict the actual values, as shown in Figure 2.11 and 2.12, is because of the significant difference in variance discussed in Section 2.2.

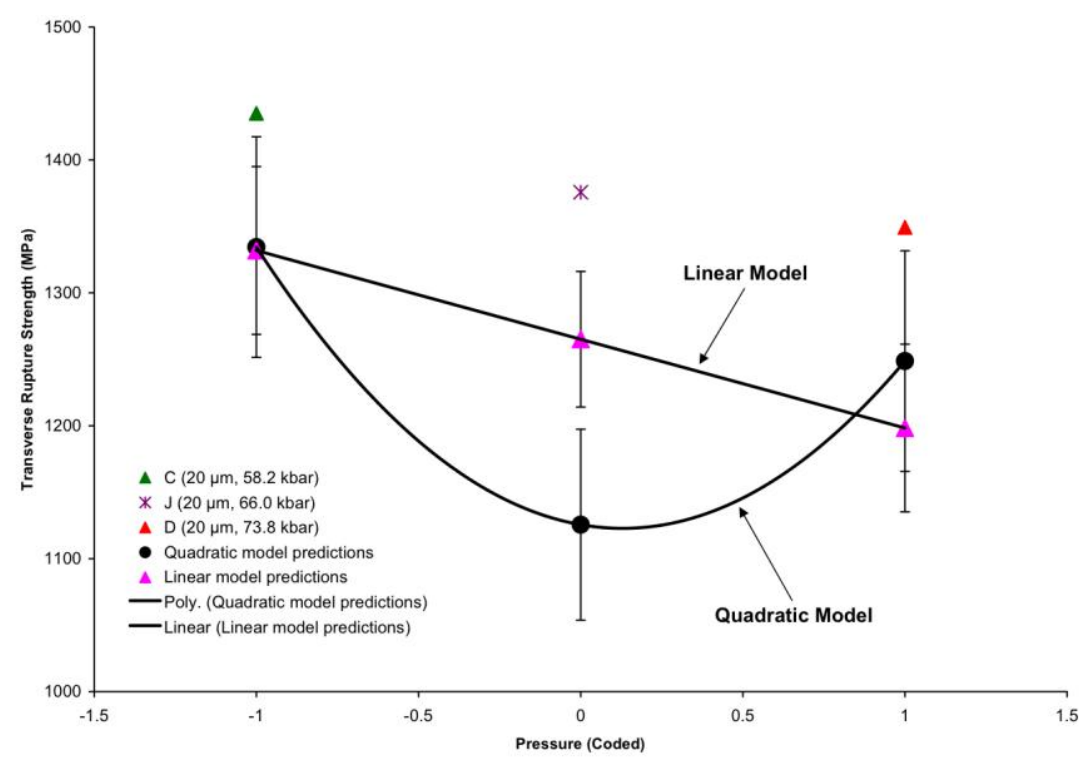

Figure 2.12: Average values of $\mu_{2}$ for designs $\mathrm{C}, \mathrm{D}$ and $\mathrm{J}$ compared to the linear and quadratic model predictions

The response surface models of equations (2.4) and (2.16) are shown in Figure 2.13 for comparison. We expect equation (2.16) to better approximate the relationship between $\mu_{2}$ and the design variables, and should therefore lead to a more adequate Pareto frontier model. 

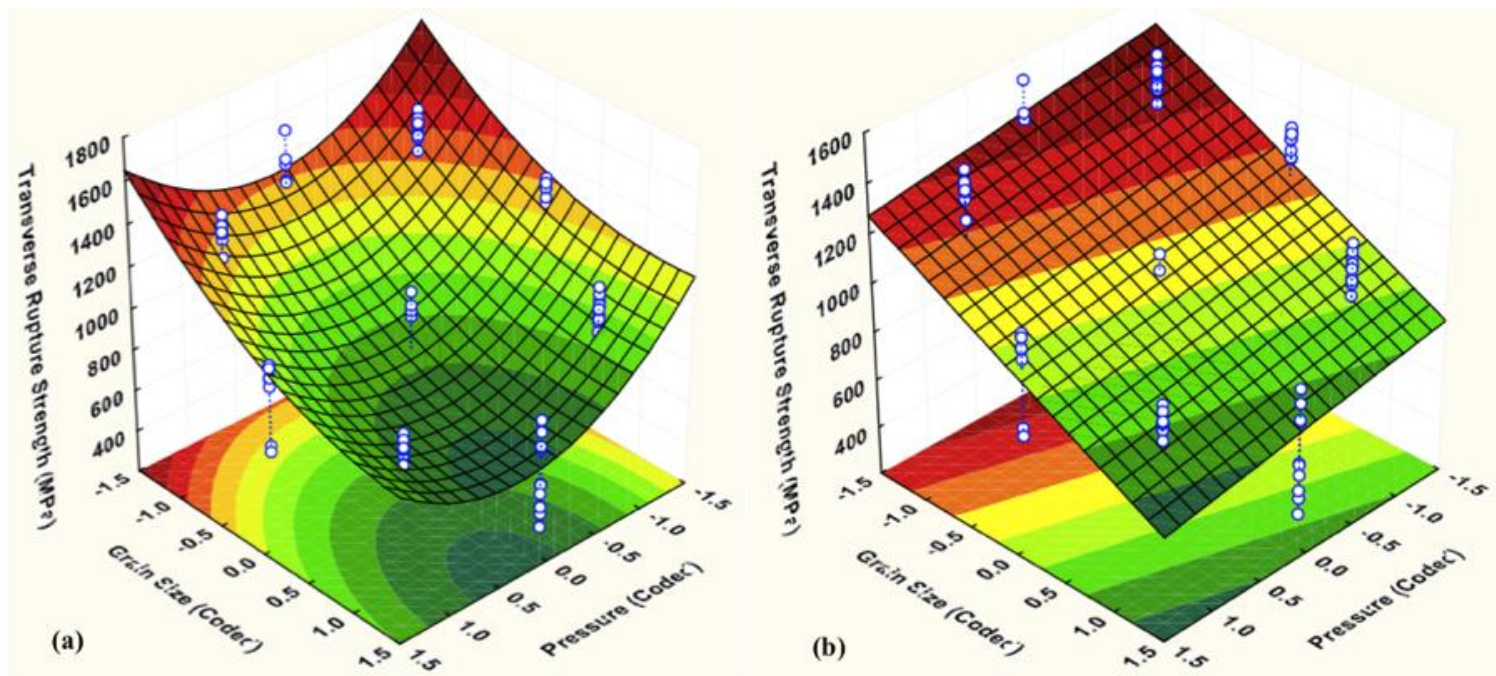

Figure 2.13: Comparison of the quadratic (a) and linear (b) response surface models for $\mu_{2}$

Using the normal constraint method with equations (2.3) and (2.16), we obtain the new Pareto frontier approximation shown in Figure 2.14. Unlike the first approximation, the new Pareto frontier is one continuous segment.

Figure 2.15 illustrates the new Pareto frontier in comparison to all of the experimental data with a $95 \%$ confidence region. It is interesting to see that all four of the additional experimental runs are Pareto optimal compared to the rest of the data. Seven of the eight non-dominated designs fall within the confidence region of this new model. 


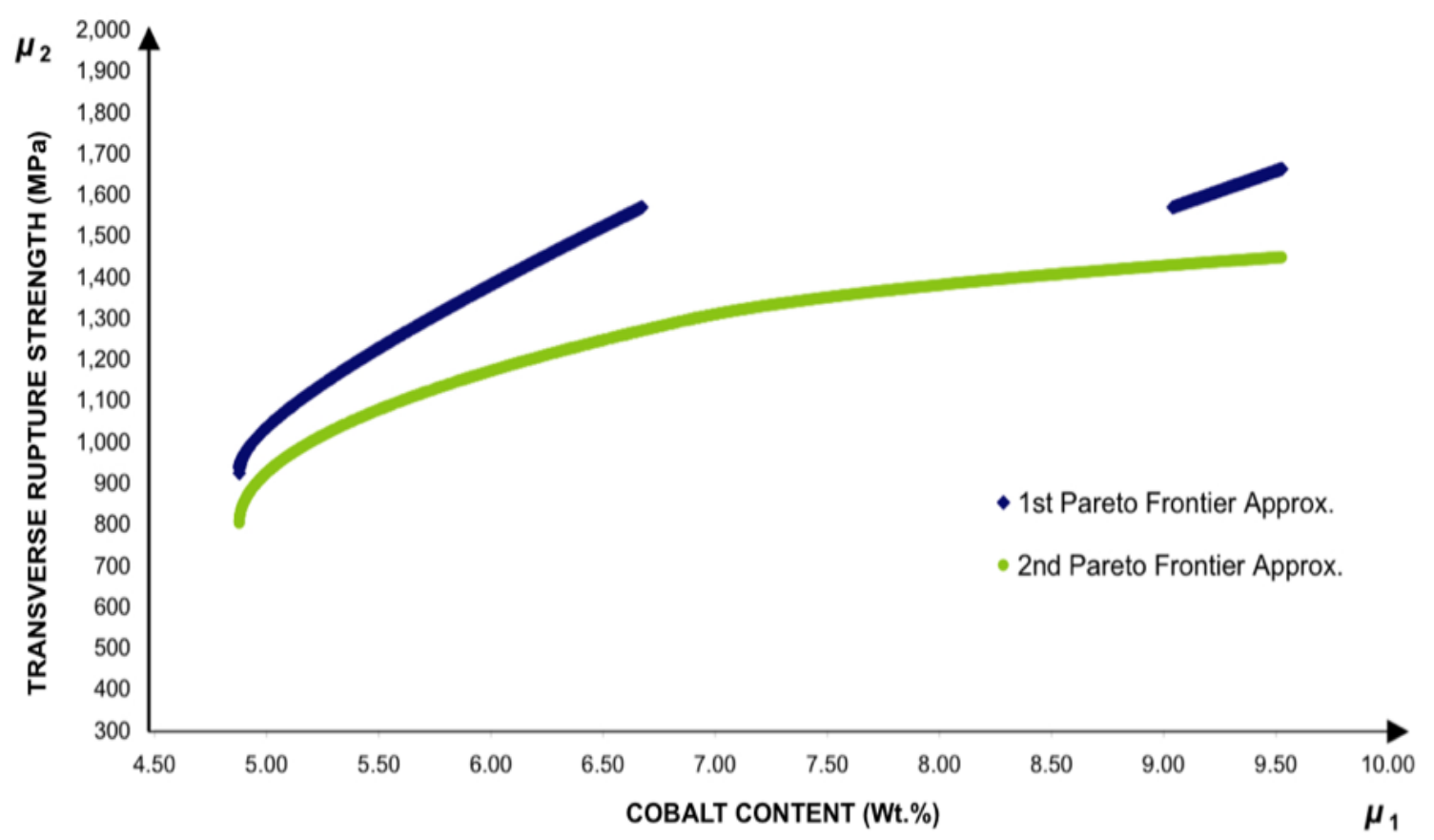

Figure 2.14. Comparison of the first and second Pareto frontier approximations

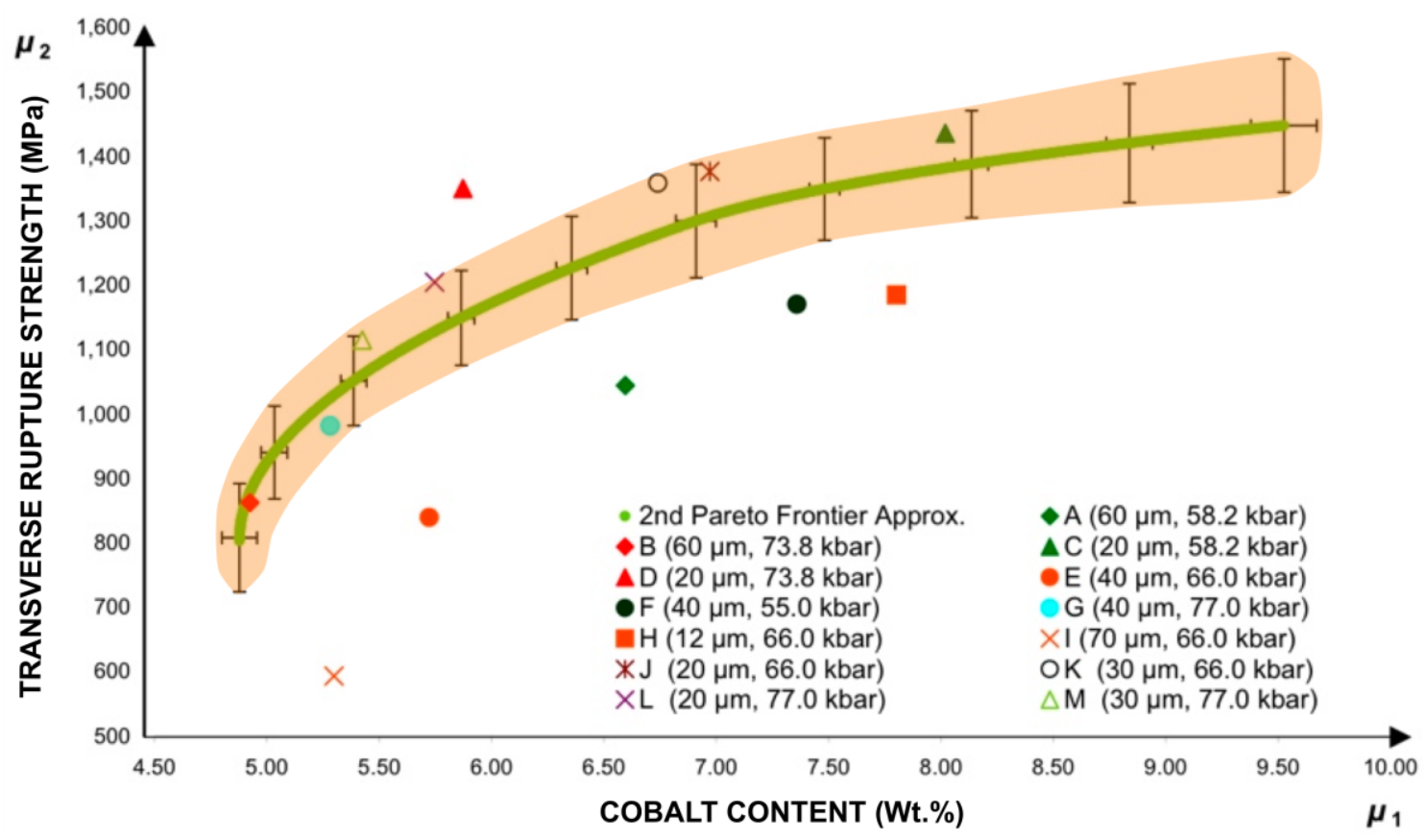

Figure 2.15: The second and final Pareto frontier model with the $95 \%$ confidence region 
As a check, the first Pareto frontier model is reproduced in Figure 2.16 with the additional data. Once again, only two of the four new designs fall within its confidence region, as shown in Figure 2.16. Although the second Pareto frontier approximation does not completely encompass all of the non-dominated designs, it provides a better approximation than the first, as measured by the number of designs that fall within its confidence region.

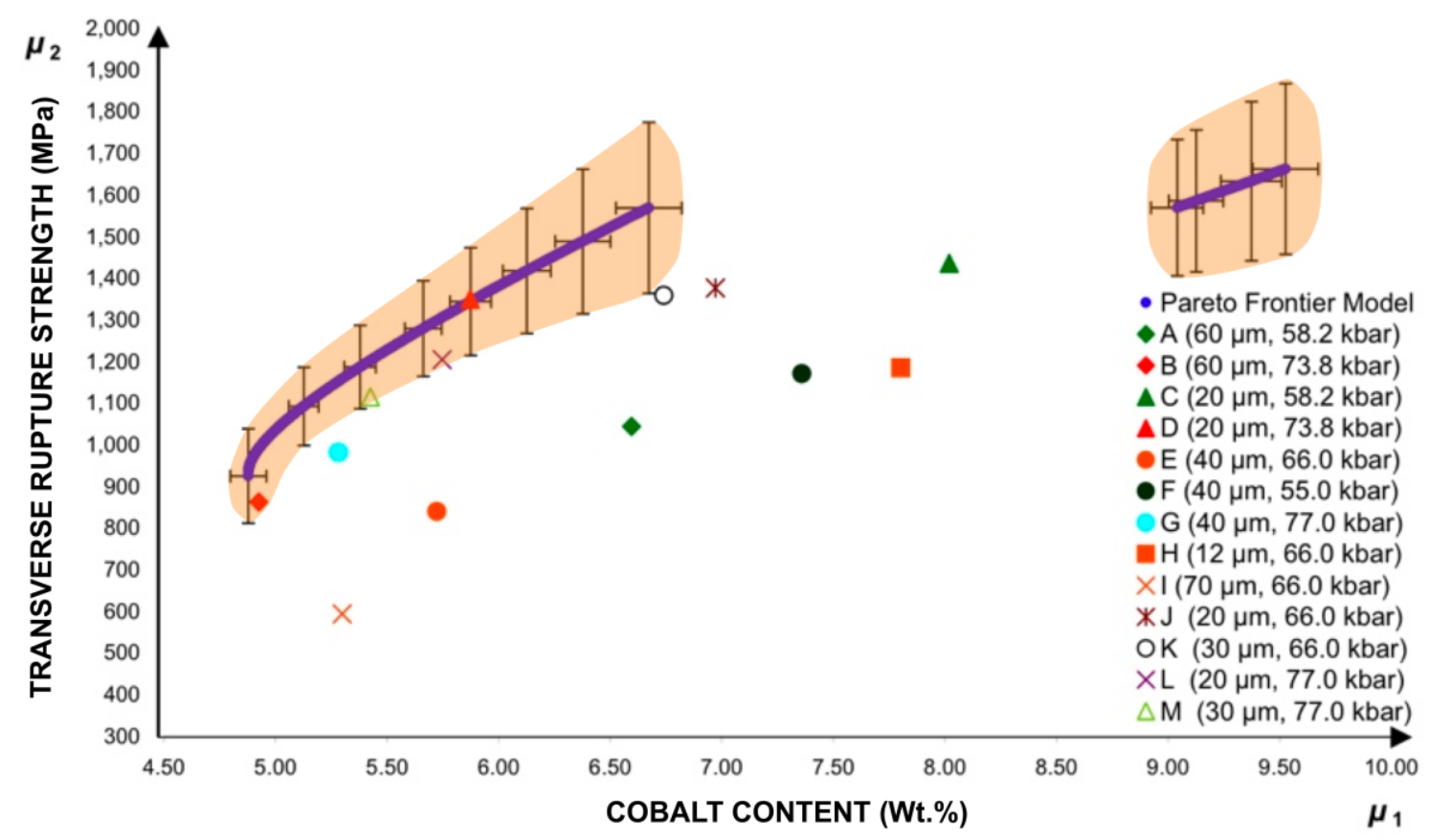

Figure 2.16: The first Pareto frontier model plotted against all 13 experimental runs

Finally, from a design standpoint, we would like to know what combinations of design variables produce the best designs. Figure 2.17 illustrates the new Pareto frontier model mapped to the design variable space. The greatest area of interest appears to be 
the range of pressures between 70 and $77 \mathrm{kbar}$, and grain sizes between $12 \mu \mathrm{m}$ and 55 $\mu \mathrm{m}$.

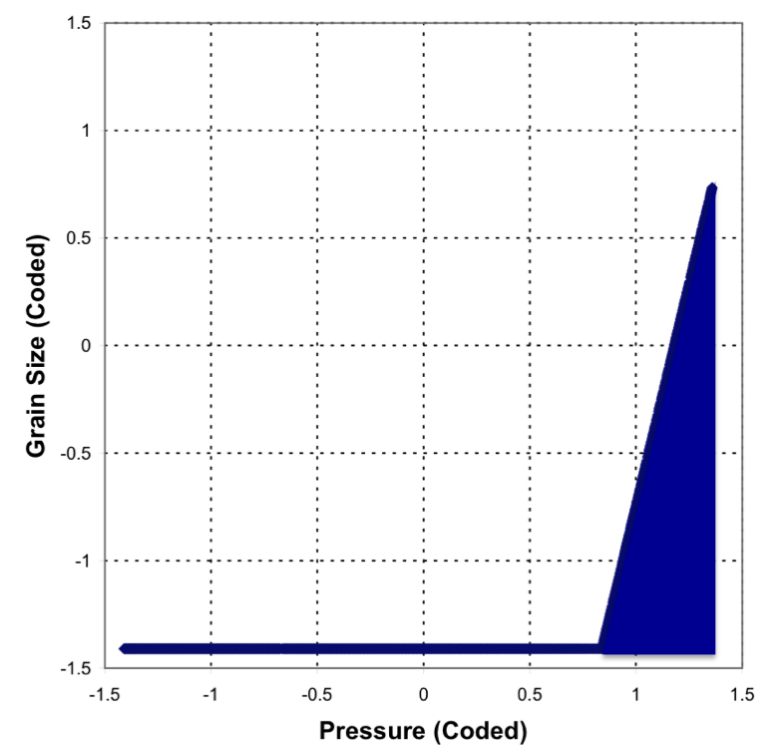

Figure 2.17: The second Pareto frontier mapped to the design variable space

In summary, the normal constraint method was used to characterize the tradeoff relationship between cobalt content $\left(\mu_{1}\right)$ and transverse rupture strength $\left(\mu_{2}\right)$ of polycrystalline diamond. The first approximation is a tradeoff curve that tends to overestimate the values of $\mu_{2}$ for the Pareto-optimal experimental data, and only captures four of the eight optimal designs within its confidence region. The second approximation is a tradeoff curve that tends to slightly underestimate the values of $\mu_{2}$, but captures seven of the eight non-dominated designs in the confidence region of the Pareto frontier. For this reason, the second Pareto frontier model was deemed adequate. 


\subsection{Characterizing the Tradeoff Between $\mu_{1}$ and $\mu_{3}$}

The emergence in recent years of a significant number of new PCD manufacturers, mostly in countries where labor costs are low, has heightened costsensitivity among PCD consumers. This increase in competition is requiring engineers and business managers to become more aware of the costs that they incur for improving the performance of their materials. Therefore, understanding the influence of manufacturing cost on the first tradeoff model developed in this study is important to its practical implementation. For this reason, we are interested in characterizing the tradeoff between cobalt content and manufacturing cost.

The characterization of the tradeoff between $\mu_{1}$ and $\mu_{3}$ is also accomplished using the normal constraint method, and is shown in Figure 2.18. The percent increase in manufacturing cost rises sharply as the value of $\mu_{1}$ drops below $5.00 \%$.

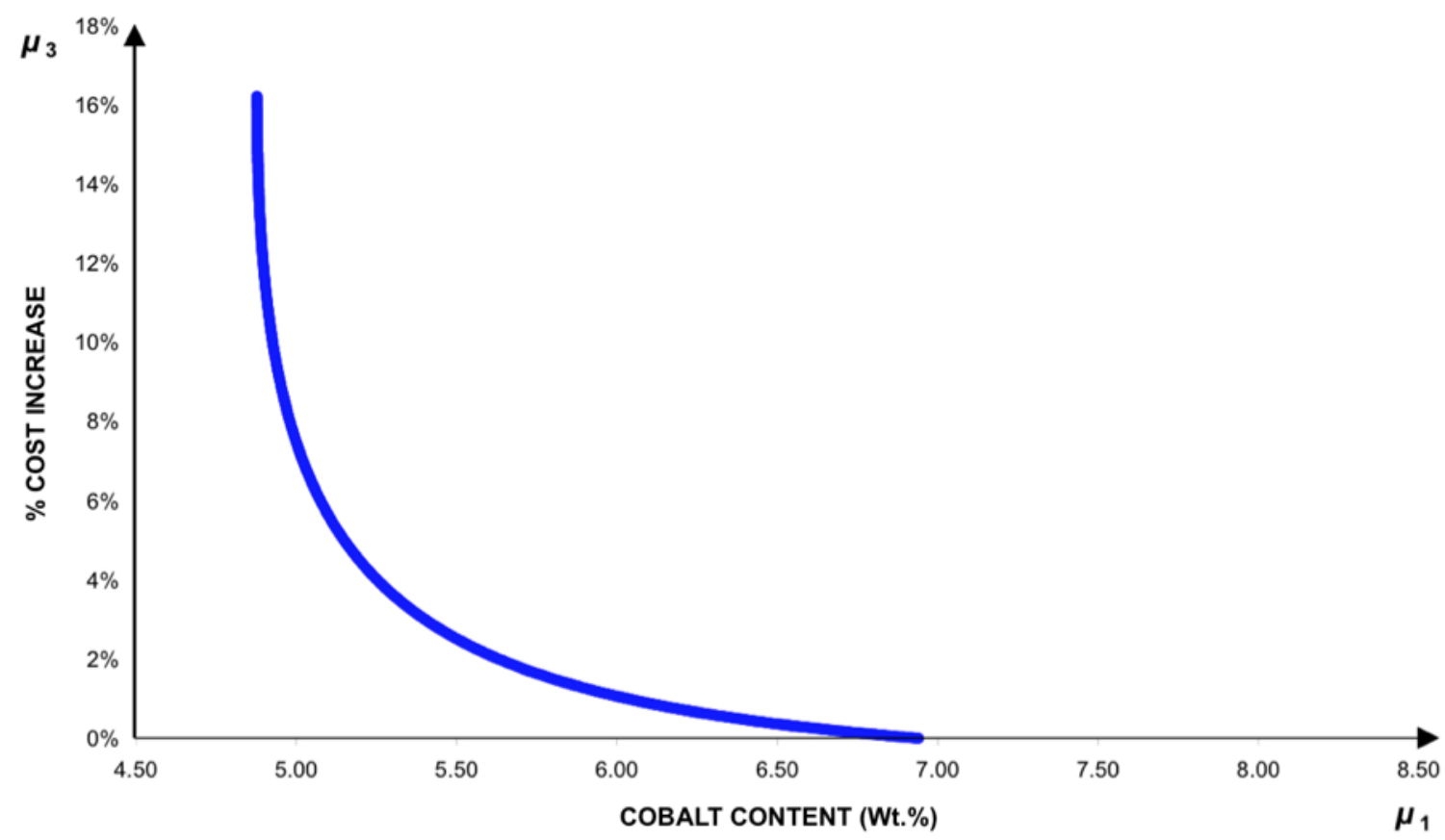

Figure 2.18: The Pareto frontier between $\mu_{1}$ and $\mu_{3}$ 
By plotting the experimental data on the same graph as the Pareto frontier in Figure 2.19, we can make several important observations. First, since manufacturing cost is only related to the sintering pressure, from equation (2.5), the experimental data falls on five distinct horizontal lines that directly correlate to the pressure at which they were processed. Second, the designer can reduce the amount of cobalt in the PCD for a given pressure by increasing the diamond grain size. This is one way that a manufacturer can potentially improve thermal stability without incurring additional cost. Third, because of the exponential nature of the cost model, an increase in pressure at the low end adds much less to the cost than the same incremental pressure increase at the high end. For instance, increasing pressure from $55 \mathrm{kbar}$ to $66 \mathrm{kbar}$ only increases the cost by $3.2 \%$. However, increasing the pressure from $55 \mathrm{kbar}$ to $77 \mathrm{kbar}$ increases the cost by $17.2 \%$. Now notice that there is very little difference in the cobalt content between designs D, E, and $\mathrm{L}$, but there is a significant difference in cost. The same is true of designs $\mathrm{G}$ and $\mathrm{I}$. The only way to decide which designs are optimal is to include the second objective transverse rupture strength. We will discuss this in Chapter 3. 


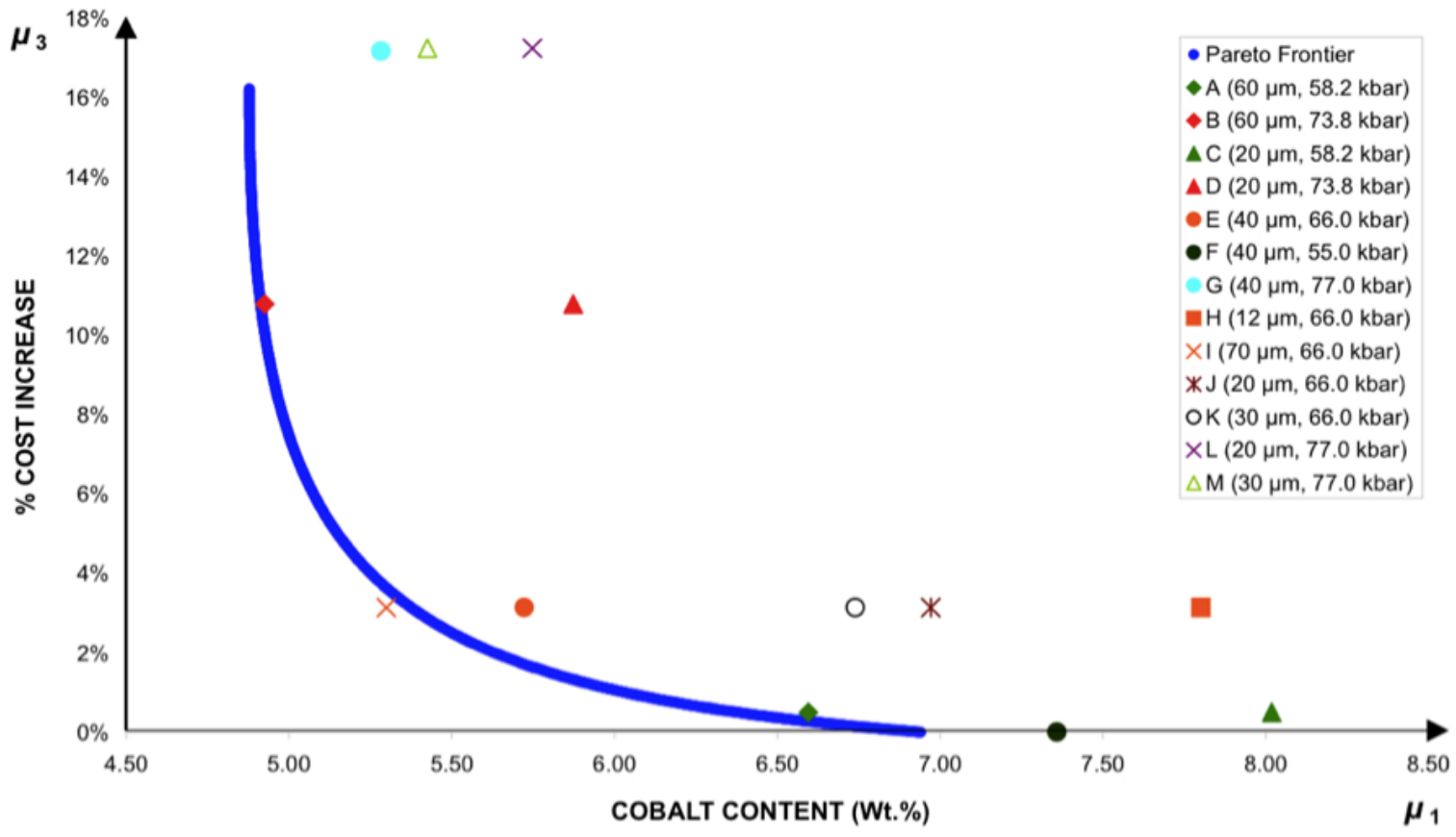

Figure 2.19: The Pareto frontier between $\mu_{1}$ and $\mu_{3}$ plotted against the experimental data 


\section{Design Evaluation and Selection Using Pareto Frontier Models}

To this point in the thesis, we have used multi-objective optimization methods to characterize the tradeoff between the competing objectives of cobalt content, transverse rupture strength, and cost of PCD. We have also briefly discussed how these tradeoff curves can be used to make design decisions. In this section, we illustrate the usefulness of the tradeoff curves in selecting the optimal PCD designs. We then validate the optimal designs by evaluating their performance in rock cutting tests.

\subsection{Selection of the Optimal PCD Design}

In the last chapter we observed that seven of the eight Pareto optimal designs from the first tradeoff model were all pressed at $66 \mathrm{kbar}$ and above. The second tradeoff model showed that developing designs at $66 \mathrm{kbar}$ and above will incur the largest cost increase. What we lacked in that discussion was a visual representation of how the transverse rupture strength influences manufacturing cost.

We did not develop a tradeoff model between $\mu_{2}$ and $\mu_{3}$ because when using equations (2.4) and (2.5) in the normal constraint method, the best design for $\mu_{2}$ is also the best design for $\mu_{3}$. This means that no tradeoff exists between these objectives, and it makes sense because lower sintering pressures (i.e. lower costs) produce higher values of transverse rupture strength for a given diamond grain size. 
Even with this lack of a tradeoff, it is still helpful to plot the values of $\mu_{1}, \mu_{2}$, and $\mu_{3}$ of the experimental data all on one plot as shown in Figure 3.1. The plot in Figure $3.1 \mathrm{~b}$ is the same data as in $3.1 \mathrm{a}$, only shown at a slightly different perspective in order to provide more clarity. The designs located in the shaded volume are all Pareto optimal designs in the $\mu_{1}-\mu_{2}$ plane. The three designs grouped at the very top part of the shaded region in Figure 3.1b happen to be the most expensive designs to manufacture because they are all pressed at 77 kbar. The two designs just below this group also fall along the $\mu_{1}-\mu_{2}$ Pareto frontier and are the second most costly Pareto designs. These designs are likely to be the best compromise considering all three objectives.
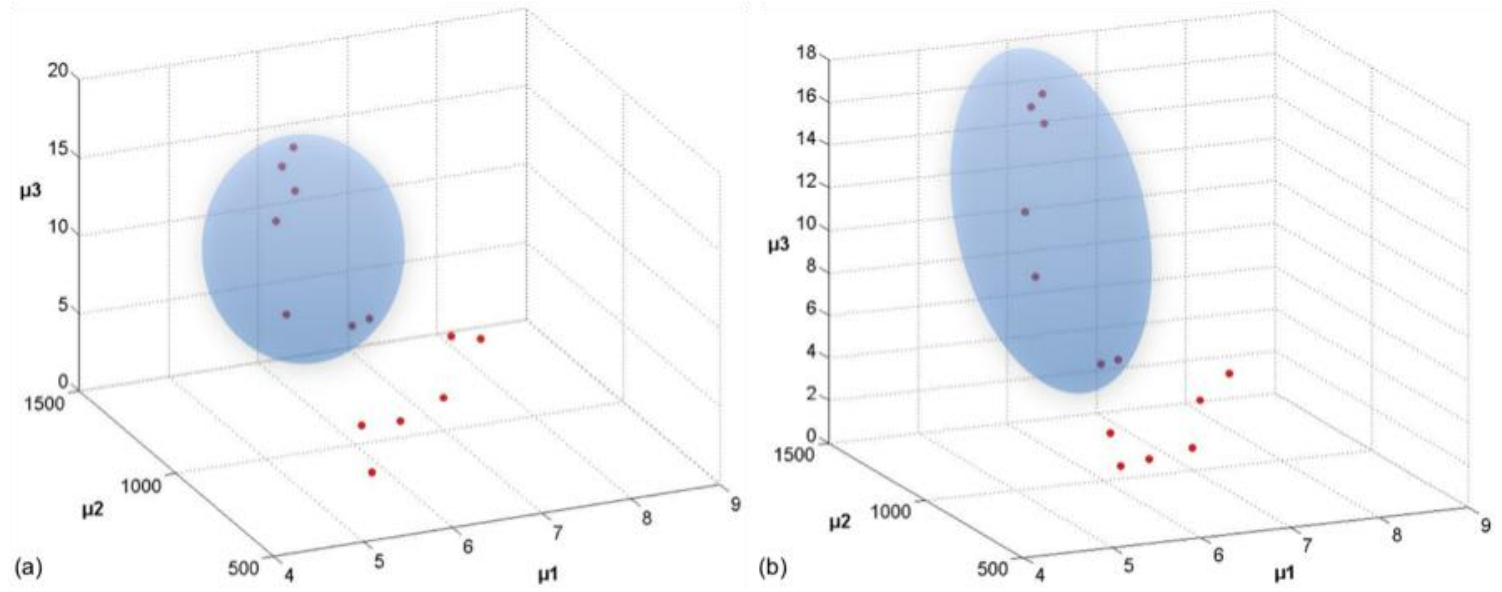

Figure 3.1: Three-dimensional plots of $\mu_{1}, \mu_{2}$ and $\mu_{3}$

Imagine that all of the red dots from Figure 3.1 are translated straight down onto the $\mu_{1}-\mu_{2}$ plane. This is what Figure 3.2 represents. Eight of the 13 experimental designs shown in the figure are Pareto optimal (we consider design $\mathrm{D}$ to be Pareto optimal because it is obviously a Pareto design in relation to the rest of the experimental data). An infinite number of Pareto optimal designs are possible, but we will only focus 
on designs that have been built and tested. Notice the shaded ellipse surrounding five of the designs. These designs are highlighted in red because they represent the best compromise in terms of both objectives. To narrow things down even further, notice how there is not a significant difference in $\mu_{2}$ between designs $\mathrm{D}$, J, and $\mathrm{K}$. The optimal design of these three is $\mathrm{D}$. Also, there is not a significant difference in $\mu_{1}$ between designs $\mathrm{D}$ and $\mathrm{L}$, but there is a noticeable difference in $\mu_{2}$. It seems that from this perspective, design D offers the best tradeoff between cobalt content and strength. Now let us look at manufacturing cost.

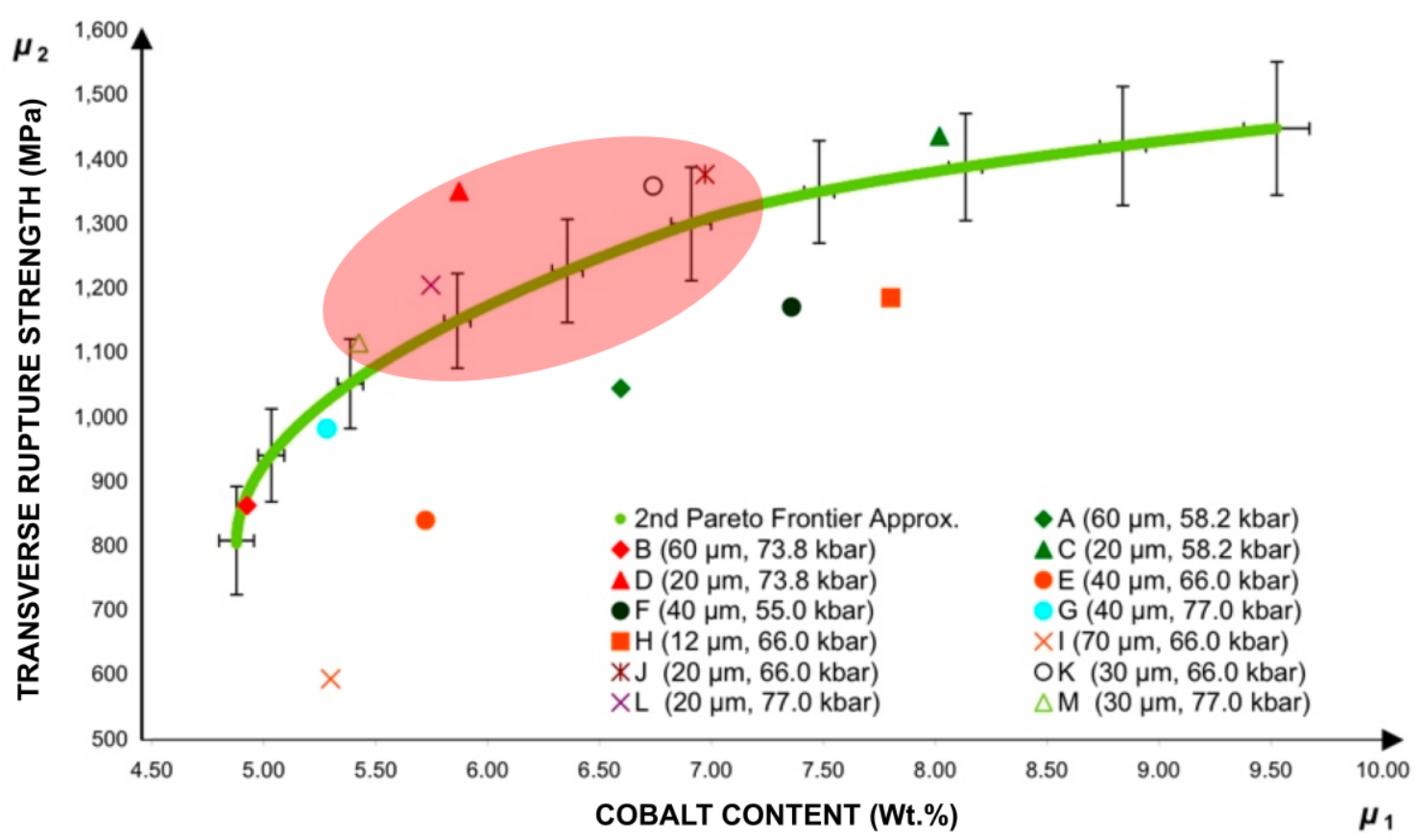

Figure 3.2: The tradeoff characterization for $\mu_{1}$ and $\mu_{2}$

The three designs highlighted by the blue circle in Figure 3.3 are designs G, L, and M. The latter two are considered to be two of the five optimal designs. However, these two designs are also the most costly to manufacture. Since neither L nor M was 
considered the optimal design in terms of $\mu_{1}$ or $\mu_{2}$, we will exclude them both from consideration at this point. The two designs highlighted in the orange oval are the next most expensive designs. The design with the arrow pointing to it is design $\mathrm{D}$, which was found to be the optimal design in terms of the first two objectives. The fact that $\mathrm{D}$ is not the most expensive design to manufacture also increases its desirability.

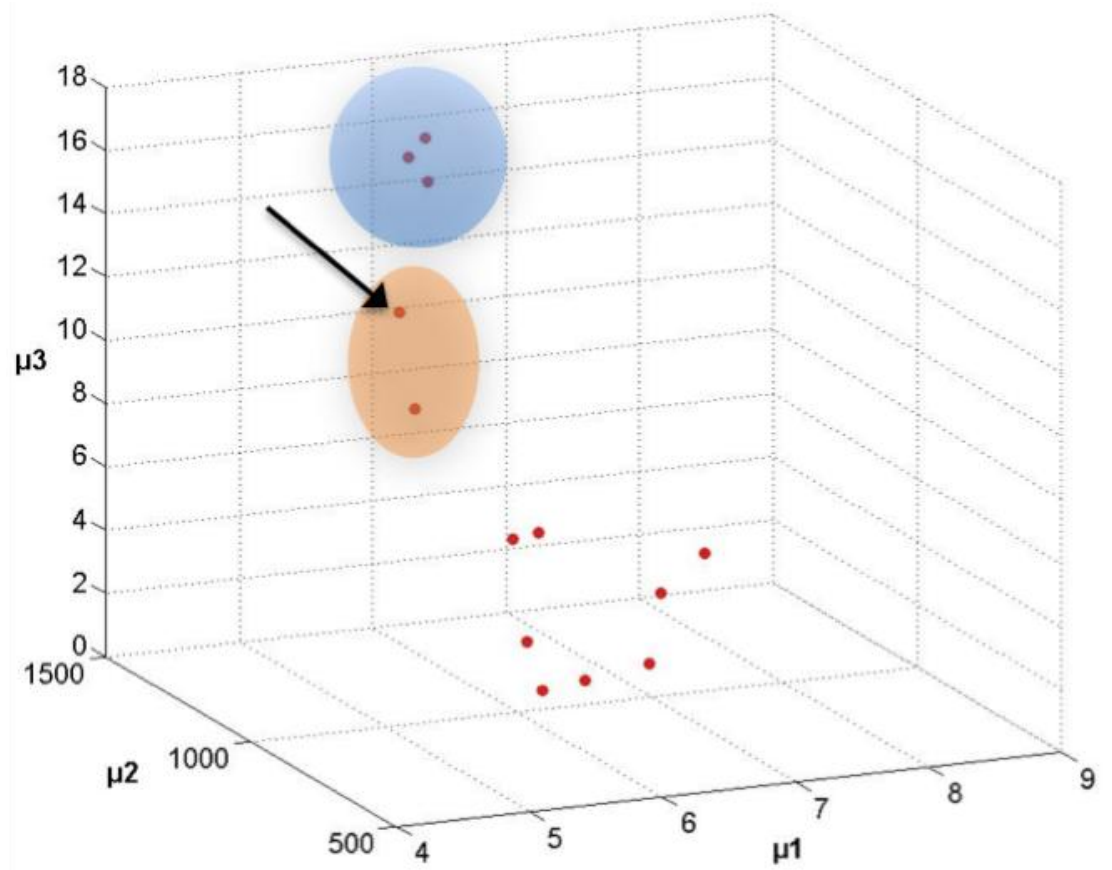

Figure 3.3: Three-dimensional plot of $\mu_{1}, \mu_{2}$ and $\mu_{3}$ showing the most "optimal" PCD design, $\mathrm{D}$ (indicated by the arrow)

Now that we have decided that design $\mathrm{D}$ is the is the most desirable design in terms of all three objectives, we will evaluate this design against the others in cutting tests to validate this choice. 


\subsection{Evaluation of the Optimal PCD Design}

We have assumed that tensile strength and cobalt content (i.e. thermal-resistance) are important properties related to wear, and that characterizing the tradeoff between them will help lead to the design of more wear-resistant parts. To check this assumption, the PCD designs A - I were subjected to rock cutting tests, specifically to see how the wear-resistance of designs $\mathrm{D}, \mathrm{J}, \mathrm{K}, \mathrm{L}$, and $\mathrm{M}$ compared with the rest of the designs.

A common method for testing the wear rate of PCD in the drilling industry is to mount a PCD compact in a vertical turret lathe and machine a piece of granite. A picture of the apparatus we used for the tests is shown in Figure 3.4. The volume of rock removed per unit of diamond worn away is typically used as a measure of the wear and is referred to as the grinding ratio (G-ratio). Rock cutting tests were conducted on PCD compact samples that were prepared at the same manufacturing conditions as groups A I, only the diamond layers were left attached to the tungsten carbide substrate.

Two tests were carried out using the cutting parameters listed in Table 3.1. Test I was designed to emphasize actual drilling conditions of a PCD compact in service on a drill bit. Test II was designed to put a significant thermal load on the diamond, which eventually caused the diamond to graphitize and stop cutting. The linear distance traveled by the cutter before burning up was used as the measure in Test II and will be referred to as the distance-to-failure. This test is a measure of the thermal resistance of the PCD. 


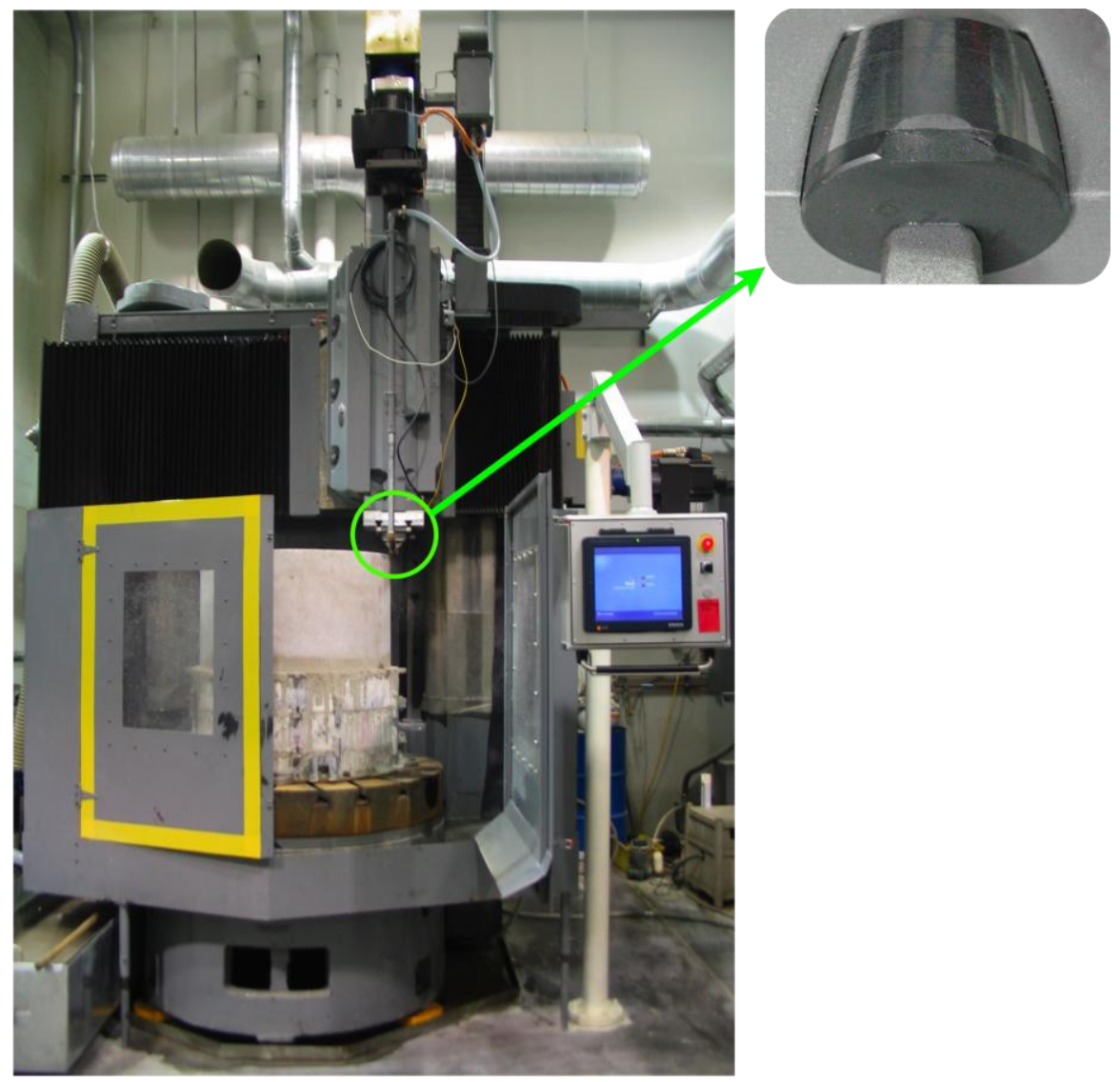

Figure 3.4: Vertical turret Lathe apparatus used for testing the wear rate of PCD

Table 3.1: Rock cutting parameters for Test I and Test II.

\begin{tabular}{cccccc}
\hline Test & $\begin{array}{c}\text { Depth of } \\
\text { Cut }(\mathbf{m m})\end{array}$ & $\begin{array}{c}\text { INFEED } \\
(\mathbf{m m} / \mathbf{r e v})\end{array}$ & RPM & $\begin{array}{c}\text { SURFACE } \\
\text { SPEED }(\mathbf{m} / \mathbf{s})\end{array}$ & COOLANT \\
\hline I & 0.254 & 6.35 & 101 & Variable & Yes \\
II & 1.270 & 1.524 & Variable & 21.3 & No \\
\hline
\end{tabular}

To analyze the results, a response surface was generated from the results of each rock cutting test in terms of the design variables. Contour plots of these surfaces are illustrated in Figure 3.5 (Test I) and Figure 3.6 (Test II). The shaded region in each of 
these figures represents the Pareto frontier mapped to the design variable space, as shown previously in Figure 2.17.

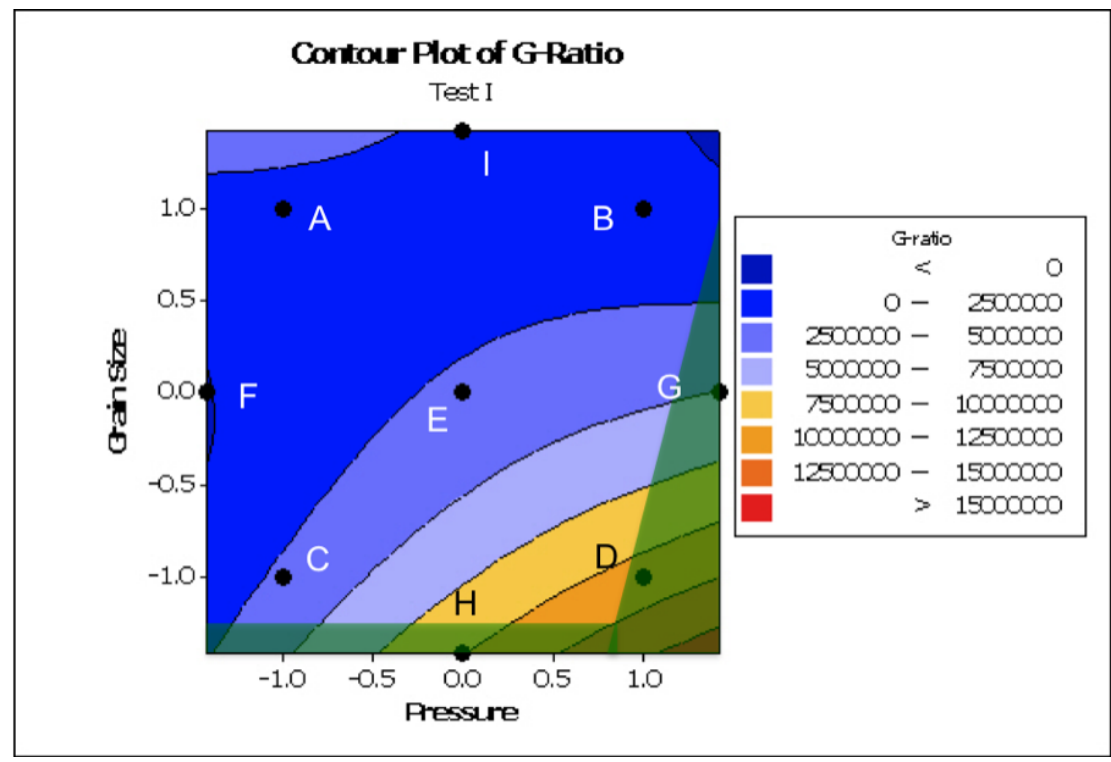

Figure 3.5: Contour plot of the G-ratio for designs A - I (Test I), with Pareto frontier highlighted

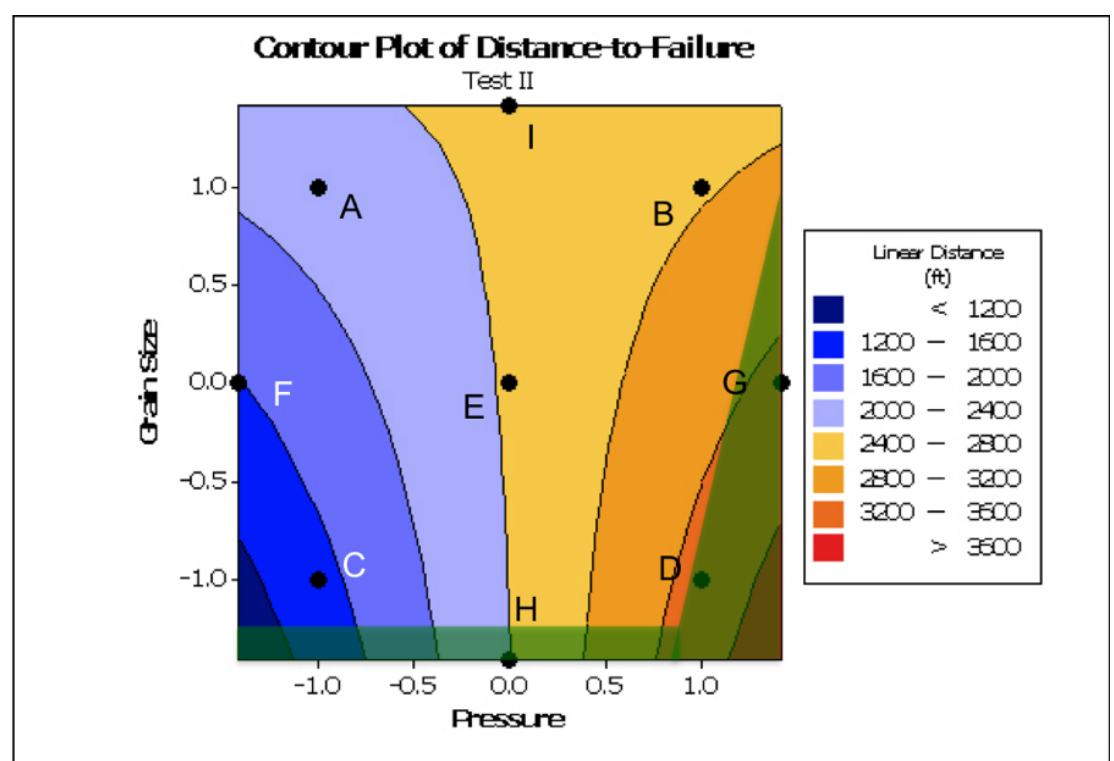

Figure 3.6: Contour plot of distance-to-failure for designs A - I (Test II) 
These rock cutting results show a correlation between the Pareto frontier and the wear-resistance of PCD. Figure 3.5 shows that the maximum G-ratio occurs in the region where sintering pressure is high and where diamond grain size is small. Figure 3.6 also shows that the maximum distance-to-failure occurs in this area. This region corresponds to the portion of the Pareto frontier where transverse rupture strength is relatively high, and where cobalt content is low. In other words, the designs with the best compromise between these material properties are also the designs that maximize the wear-resistance of the PCD. Design D had the highest wear-resistance. This correlation is important because it provides some validation for the Pareto frontier model.

Another important implication of this correlation is that it provides support for the assumption made in the beginning that cobalt content is a good substitute measure for thermal-resistance. With respect to rock cutting under the conditions described in Table 3.1, the distance-to-failure is a meaningful representation of thermal-resistance because it measures the point at which the diamond graphitizes and becomes useless as a cutting tool. Figure 3.6 clearly shows that an increase in sintering pressure is desirable for extending the distance-to-failure for a fixed grain size. This result correlates very well with the response surface model in Figure 2.4, which shows that the cobalt content decreases as sintering pressure increases for a fixed grain size.

With this validation from the rock cutting results, the Pareto frontier is useful to designers because it quantifies how much a designer must "give up" in strength in order to gain in thermal-resistance. It also implies that further improvements to the wearresistance and thermal-resistance can be made by increasing pressure and decreasing grain size. Having a reliable correlation between the wear-resistance of PCD and its 
material properties gives the designer a way to gauge the relative cutting performance of a new design without necessarily going to the expense of running wear tests. Also, the $\mu_{1}$ - $\mu_{2}$ model in combination with the $\mu_{1}-\mu_{3}$ model shows that the best performing designs come at a price. This information can also be useful for engineers and managers in new product development, because they can see where existing products lie in relation to the Pareto frontier. 


\section{Conclusion}

\subsection{Conclusions}

In this thesis, multi-objective optimization methods are brought to bear in characterizing the tradeoff relationship between cobalt content (i.e. thermal-resistance), transverse rupture strength, and manufacturing cost of polycrystalline diamond.

In order to accomplish this, a process is developed that combines response surface methodology with the normal constraint method in an iterative fashion to generate Pareto frontiers between two empirically based objectives. This method is summarized in the following three-step process:

(1) Determine the objectives of interest and establish an experimental program to develop the empirical models for each objective.

(2) Gather the data and build the models for each objective, or refine the models of interest.

(3) Generate the Pareto frontier using the normal constraint method, establish

a confidence region around the Pareto frontier, and check the adequacy of the Pareto frontier against a set of experimental data. Repeat Steps 2 and 3 if needed. This method can be adapted to situations where the empirical models for an objective are well established or where they are not yet discovered. The confidence region is established around the Pareto frontier by incorporating statistical confidence limits on the 
Pareto solutions. This is an essential part of this method because it allows the designer to gauge whether experimental designs are likely to be true Pareto designs or not, and therefore provides a way to measure the adequacy of a Pareto frontier model.

In the process of characterizing the Pareto frontier, empirical relationships are developed between the objectives of cobalt content and transverse rupture strength and the design variables of sintering pressure and initial diamond grain size. The relationship for cobalt content shows that sintering pressure can be used to alter the cobalt content of PCD for a given grain size, and that as pressure is increase, the cobalt content decreases.

The Pareto frontier model for cobalt content and transverse rupture strength is non-linear. As cobalt content drops below approximately 5\%, the decrease in transverse rupture strength becomes more severe. There is relatively little change in the transverse rupture strength of Pareto optimal designs once the cobalt content reaches approximately 7.5\%. The best Pareto optimal designs occur at grain sizes of $20 \mu \mathrm{m}$ and $30 \mu \mathrm{m}$, and at sintering pressures between $66 \mathrm{kbar}$ and $77 \mathrm{kbar}$. Of the experimental designs built and tested, the optimal design in terms of manufacturing cost as measured by wear tests occurs at $20 \mu \mathrm{m}$ grain size and $73.8 \mathrm{kbar}$.

The Pareto frontier model for cobalt content and manufacturing cost shows that designers can minimize the cobalt content without incurring any cost by increasing the diamond grain size for a given pressure. It also shows that improving the cobalt content objective other than by adjusting grain size requires an increase in the manufacturing cost.

Also, the assumption that characterizing the tradeoff between cobalt content, transverse rupture strength, and manufacturing cost can help engineers design better wear 
parts is validated by the wear results of rock cutting tests. Response surface models of Gratio and the distance-to-failure of the cutting tests in terms of the design variables shows a strong correlation between the best designs for rock cutting and the best designs as indicated by the Pareto frontier models.

Finally, this thesis provides design engineers, engineering managers, and business managers with a set of models that can be used to compare the relative performance of new PCD designs without going to the expense of building and testing samples. And the models developed here provide a foundation for successful new product development in an increasingly competitive environment.

\subsection{Future Work}

Although this thesis establishes a solid foundation for understanding some of the important design tradeoffs in PCD, future work should include the following:

(1) Characterization of the tradeoff between fracture toughness and transverse rupture strength $[2,3,11]$.

(2) Efforts to further improve the adequacy and accuracy of the Pareto frontier model between cobalt content and transverse rupture strength. These efforts should largely include reducing the variability in the transverse rupture strength measurements.

(3) Developing a relationship that explicitly relates thermal stability to cobalt content and can be applied generally to the PCD industry.

(4) Continuing to apply multi-objective optimization concepts to the models developed in this thesis; for example, constructing and implementing a smart filter for eliminating the designs of relatively insignificant tradeoff [23]. 
(5) Evaluating the Pareto designs in terms of other industrial applications, such as wire-drawing, bearing surface applications, and cutting tools, to see if these models apply to more than rock cutting.

These activities would greatly improve the application and understanding of the models and methods established in this thesis. 


\section{References}

[1] Hertzberg, R. W., Deformation and Fracture Mechanics of Engineering Materials, Chapt. 10, p. 406. John Wiley \& Sons, Inc., fourth edition, 1996.

[2] Lammer, A. (1988) "Mechanical properties of polycrystalline diamonds", Materials Science and Technology, 4, 949-955.

[3] D. Miess and G. Rai (1996) "Fracture toughness and thermal resistance of polycrystalline diamond compacts", Materials Science and Engineering, A209, 270276.

[4] Fang, Z. Z. (2005) "Correlation of transverse rupture strength of WC-Co with hardness", International Journal of Refractory Metals and Hard Materials, 23, 119-127.

[5] Mattson, C. A. and Messac, A. (2003) "Concept selection using s-Pareto frontiers", AIAA Journal, 41(6), 1190-1198.

[6] Belegundu, A., and Chandrupatla, T., Optimization Concepts and Applications in Engineering, pp. 373-382, Prentice-Hall, Upper Saddle River, NJ, 1999.

[7] Deb, K., Multi-Objective Optimization Using Evolutionary Algorithms, Chapt. 1, pp. 1-12. John Wiley \& Sons, first edition, 2001.

[8] Hall, H. T. (1970) "Sintered diamond: a synthetic carbonado", Science, 169, 868-869.

[9] Bertagnolli, K. E. and Vale, R. (2000) "Understanding and controlling residual stresses in thick polycrystalline diamond cutters for enhanced durability", Finer Points (USA).

[10] Gigl, P. D. (1979) “The strength of polycrystalline diamond compacts”, in High Pressure Science and Technology, K. D. Timmerhaus and M. S. Barber, Eds. New York: Plenum, vol. 1, pp. 914-922. 
[11] Huang, B. L. et al. (1997) "Fracture toughness of sintered polycrystalline diamond (PCD)", Proceedings of the $5^{\text {th }}$ Int. Conference on Advanced Particulate Materials and Processes, MPIF Princeton, NJ, F. H. Froes \& J. D. Hebeisen, eds.

[12] Mehan, R. L. and Hibbs, L. E. (1989) "Thermal degradation of sintered diamond compacts", Journal of Materials Science, 24, 942-950.

[13] Box, G. E. P. and Wilson, K. B. (1951) "On the experimental attainment of Optimum Conditions (with discussion)", Journal of the Royal Statistical Society, Series B 13(1), pp. 1-45.

[14] Myers, R. H. and Montgomery, D. C., Response Surface Methodology: Process and Product Optimization Using Designed Experiments, Chapt. 1, p. 1. John Wiley \& Sons, Inc., second edition, 2002.

[15] Sun, L. and Wu, Q. (1999) "Transformation probability of graphite-diamond assisted by nonmetallic catalysts at high pressure and high temperature", Journal of Materials Research, 14(3), 631-633.

[16] Unpublished experimental data from US Synthetic Corp., Experimental Record \#'s 2427, 3689, 4186 (2008).

[17] Lawson, J. and Erjavec, J., Modern Statistics for Engineering and Quality Improvement, Chapt. 13, pp. 472-474, Thomson Learning, 2001.

[18] Bundy, F. P. et al. (1961) "Diamond-graphite equilibrium line from growth and graphitization of diamond", Journal of Chemical Physics, 35(2), 383-391.

[19] Roark, R. J. and Young, W. C., Formulas for Stress and Strain, Chapt. 10, McGrawHill, 1975.

[20] Messac, A. and Mattson, C. A. (2004) "Normal constraint method with guarantee of even representation of complete pareto frontier", AIAA Journal, 42, 2101-2111.

[21] Messac, A., Ismail-Yahaya, A. and Mattson, C. A. (2003) "The normalized normal constraint method for generating the Pareto frontier", Struct. Multidisc. Optim., 25, 8698.

[22] Statistica 8.0, Copyright StatSoft, Inc. (1984-2008).

[23] Mattson, C. A., Mullur, A. A., and Messac, A. (2004) "Smart pareto filter: obtaining a minimal representation of multiobjective design space", Engineering Optimization, 36(6), 721-740. 


\section{Appendix A Statistical Analysis}

This appendix provides the statistical details of the regression analysis that were used in evaluating the empirical models for cobalt content and transverse rupture stress in Chapter 2 and Chapter 3.

All regression models have some error associated with them. The error is the difference between the actual value of a measurement and the value predicted by the model. In regression analysis, the errors in the model should be as small as possible and should represent the random noise in the system. Therefore, the assumptions regarding the errors in any regression model [17] are,

(1) The errors are normally distributed

(2) The variance in the errors is constant

(3) The errors are independent

(4) The errors have a mean of zero (no bias).

This appendix provides an analysis of the errors in each of the regression models developed in this thesis. These models are equations (2.3), (2.4) and (2.16). In the case of equations (2.4) and (2.16), the errors do not necessarily adhere to the assumptions listed above. The reasons behind this are discussed in the last section of this appendix. 


\section{A.1 Normally Distributed Errors With a Zero Mean}

This section shows how well the errors from the models developed in Chapter 2 and Chapter 3 follow assumptions (1) and (4). Figures A.1, A.2, and A.3 are normal probability plots. These plots are indicative of how well the errors follow a normal distribution. They also provide some indication about the variance in the errors, which is assumption (2). Figures A.4, A.5, and A.6 are histogram plots of the errors. These plots indicate how closely the mean of the errors is to zero. They also provide a visual indication of how well the errors follow a normal distribution.

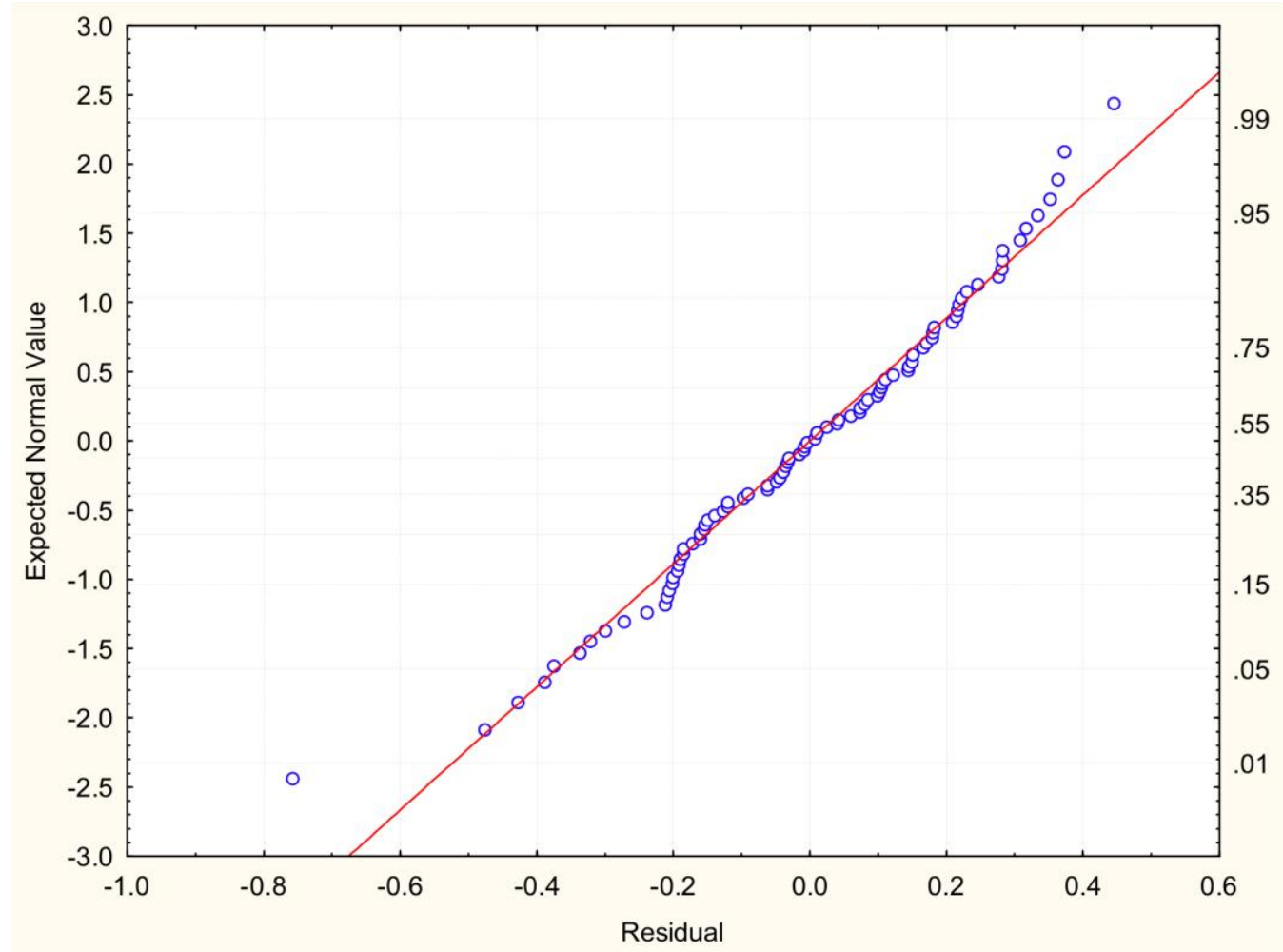

Figure A.1: The normal probability plot for the errors of equation (2.3) 


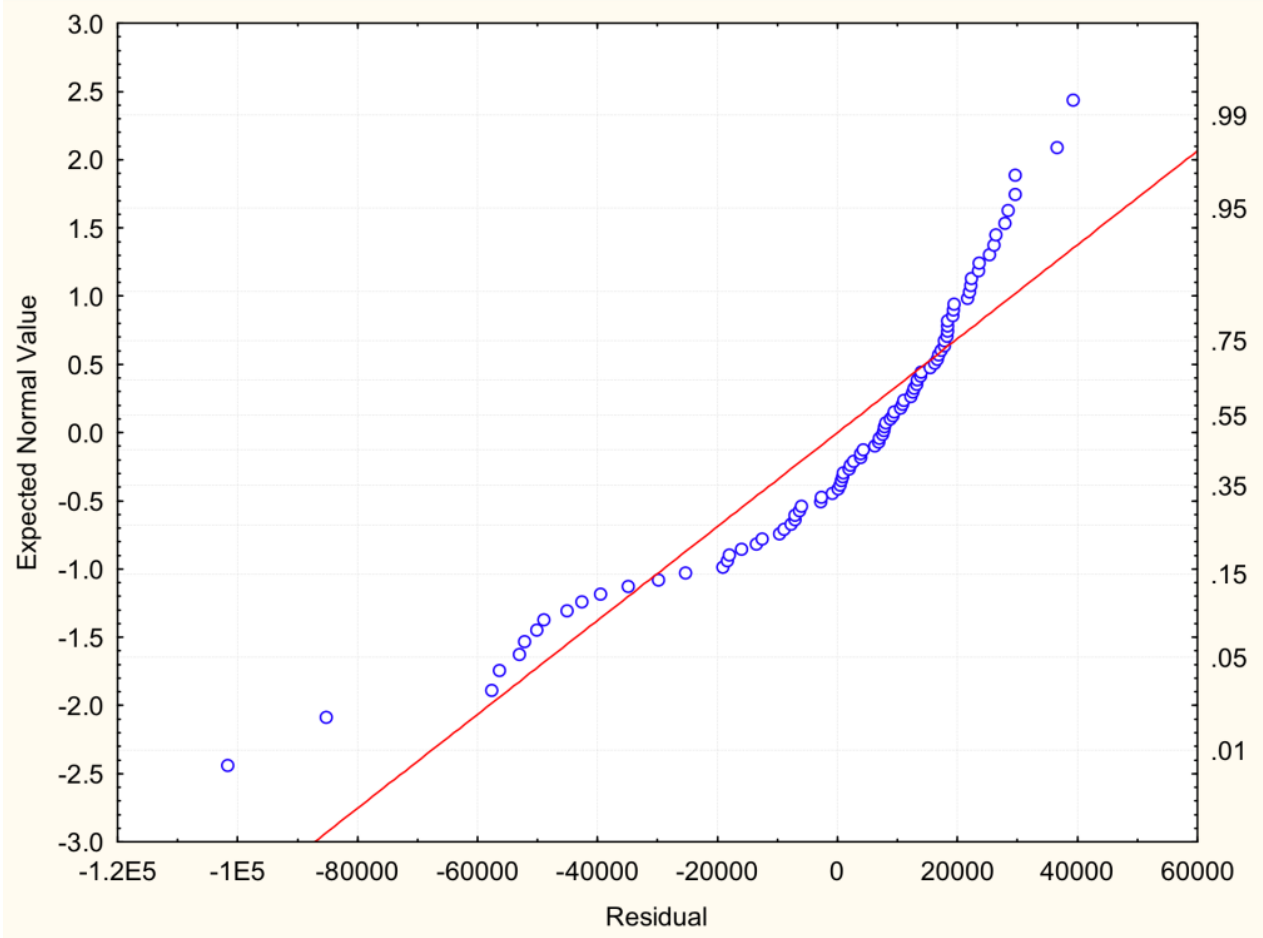

Figure A.2: The normal probability plot for the errors of equation (2.4)

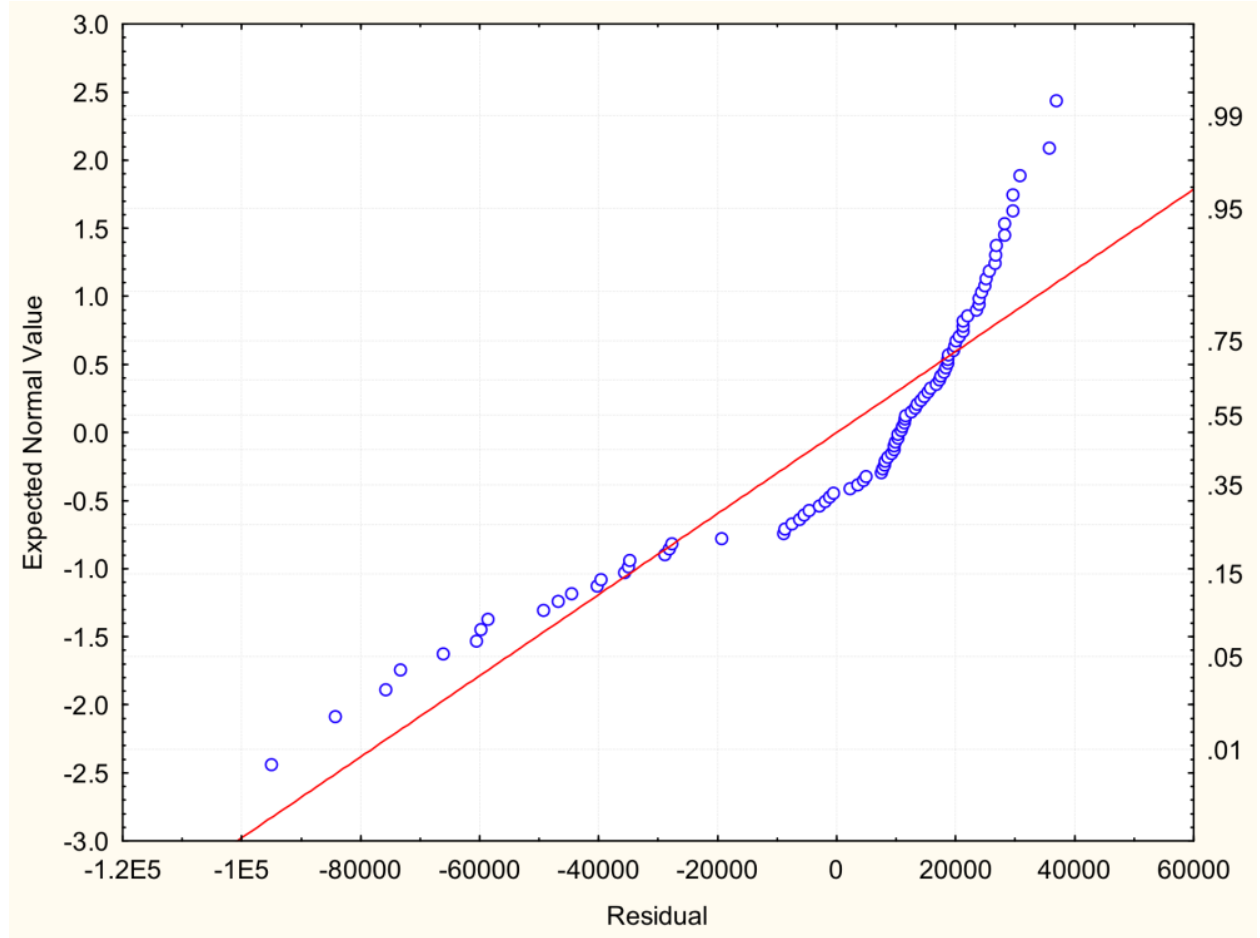

Figure A.3: The normal probability plot for the errors of equation (2.16) 


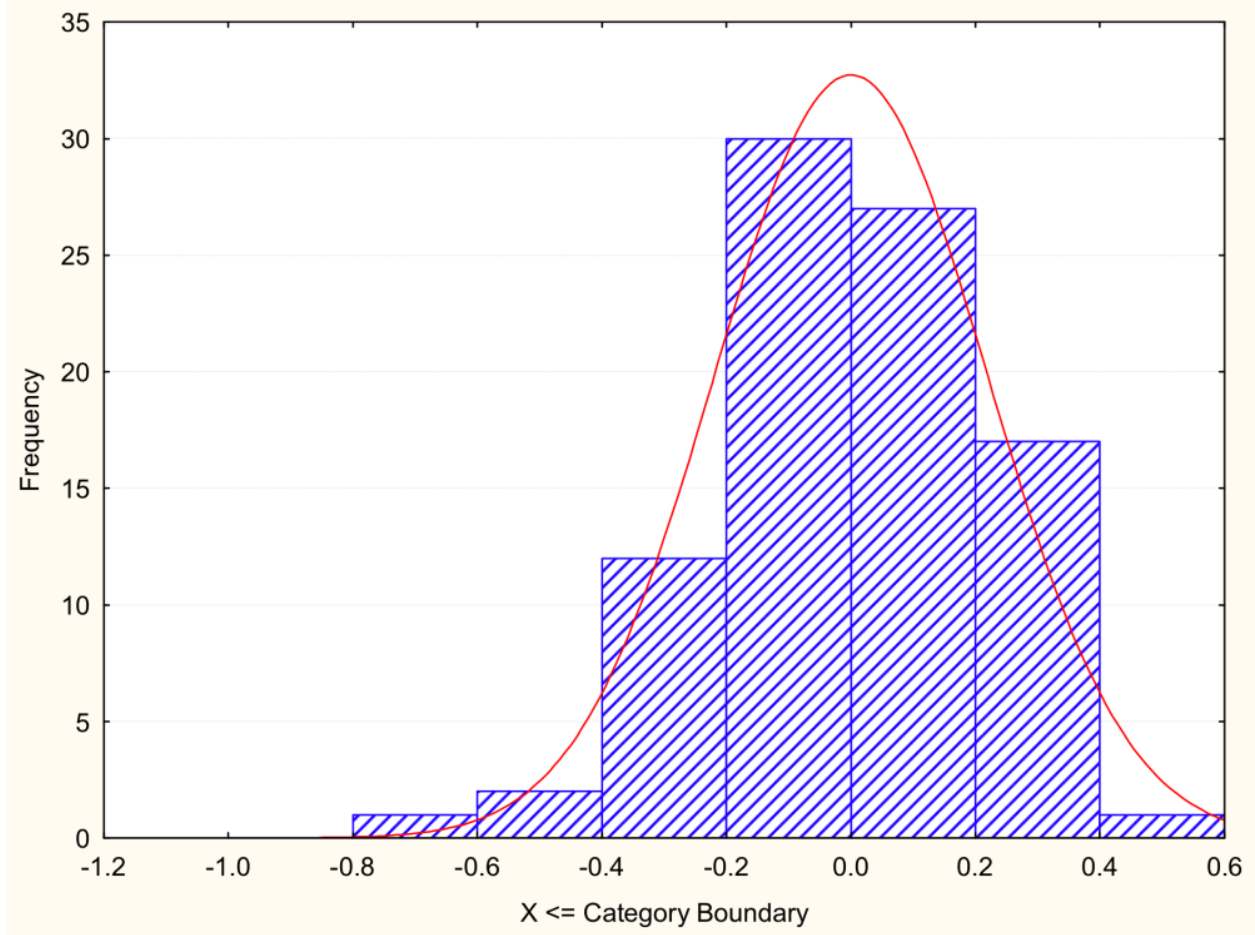

Figure A.4: A histogram of the errors of equation (2.3)

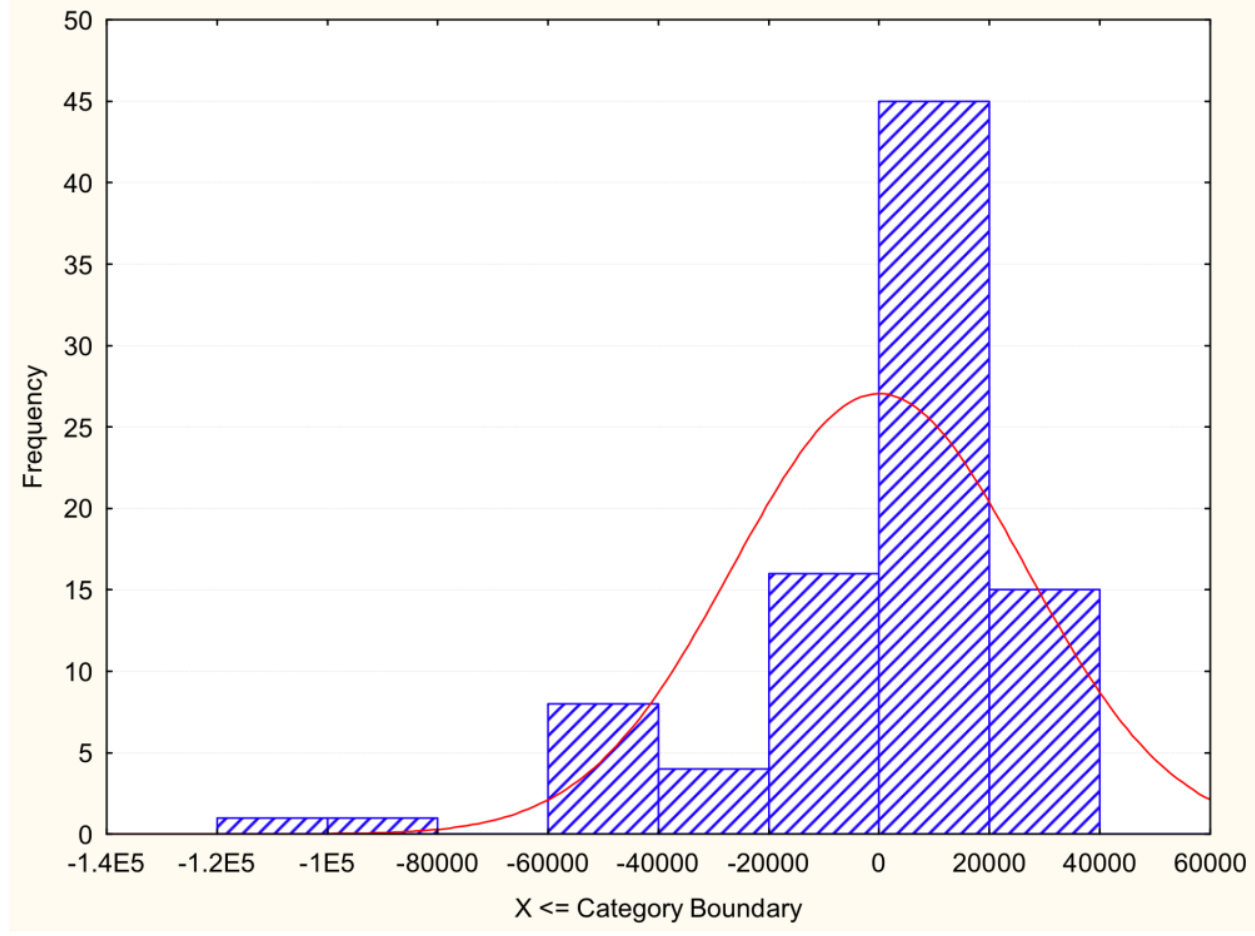

Figure A.5: A histogram of the errors of equation (2.4) 


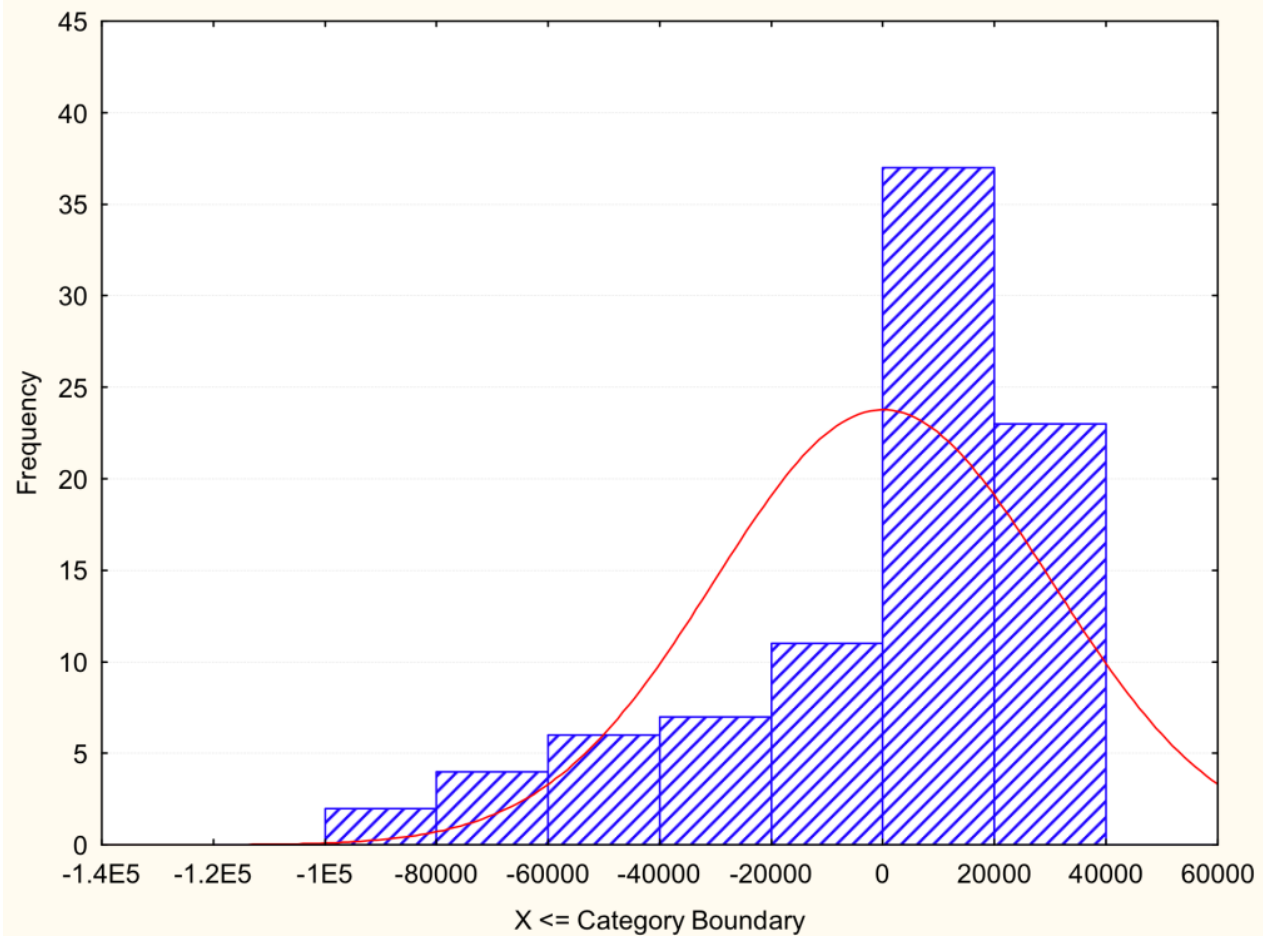

Figure A.6: A histogram of the errors of equation (2.16) showing a similar pattern to the errors of equation (2.4)

\section{A.2 Constant Variance and Independent Errors}

This section provides the analysis for assumption (2) and (3). Regression analysis assumes that the variance in the errors is constant regardless of the magnitude of the experimental measurement. It also assumes that the error of each individual measurement is independent with respect to the order in which the measurement is taken.

The third assumption relates to the idea that we want the errors to represent the random noise in the system. In order to test this assumption, the data is usually taken in a random order. However, sometimes it is not possible to take measurements in a random order. In the case of the transverse rupture strength test, we felt that running the samples in a random order could potentially risk the traceability of the data. This is because the sample labels were not readable after they were shattered. The cobalt content 
measurements were also not taken in a random order. However, the repeatability of the Koerzimat was evaluated after it had warmed up by taking measurements on a group of samples twice and observing the difference in the measured values. The mean of each set of measurements was $5.283 \%$ and $5.294 \%$, a difference of only $0.011 \%$. The standard deviations were also very similar with values of $0.088 \%$ and $0.086 \%$. This showed a high level of repeatability in the machine and provides confidence that no bias was introduced due to run order. Unfortunately, we do not have the ability to check assumption (3) for the transverse rupture strength test.

Figures A.7, A.8, and A.9 are plots of the error compared to the predicted values for each model. These plots indicate how the variance changes with the magnitude of the response. Figure A.7 shows that the variance is constant for equation (2.3). The variance for equations (2.4) and (2.16) is different between groups, as shown by Figures A.8 and A.9.

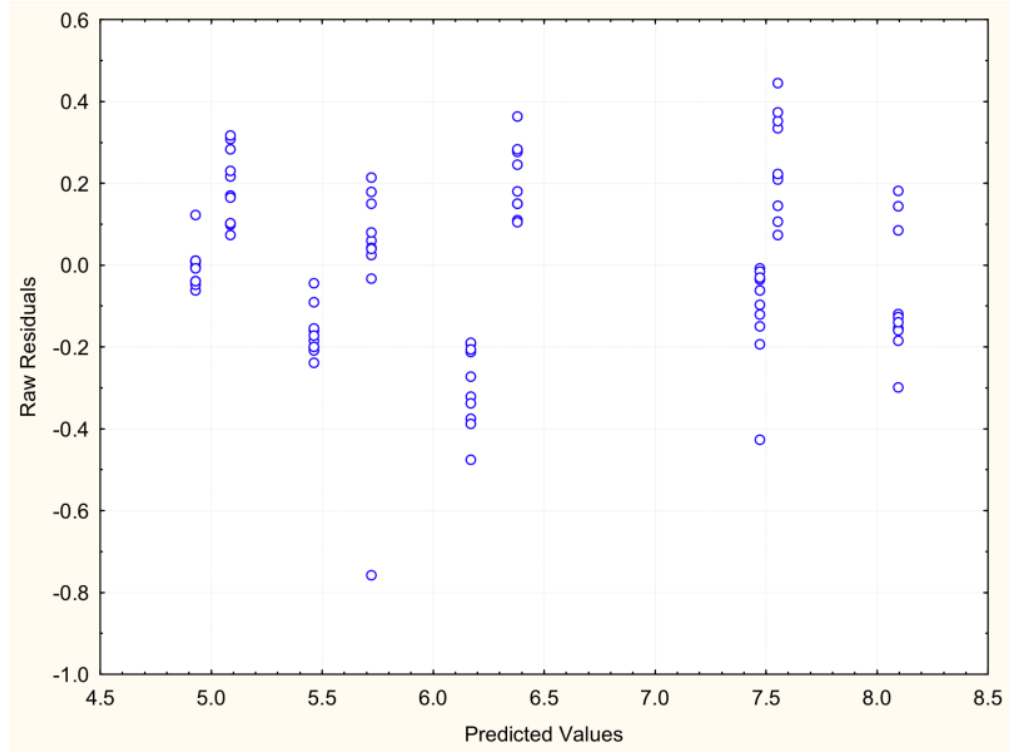

Figure A.7: A plot of the errors (vertical axis) vs. the values predicted by the model (horizontal axis) for equation (2.3) 


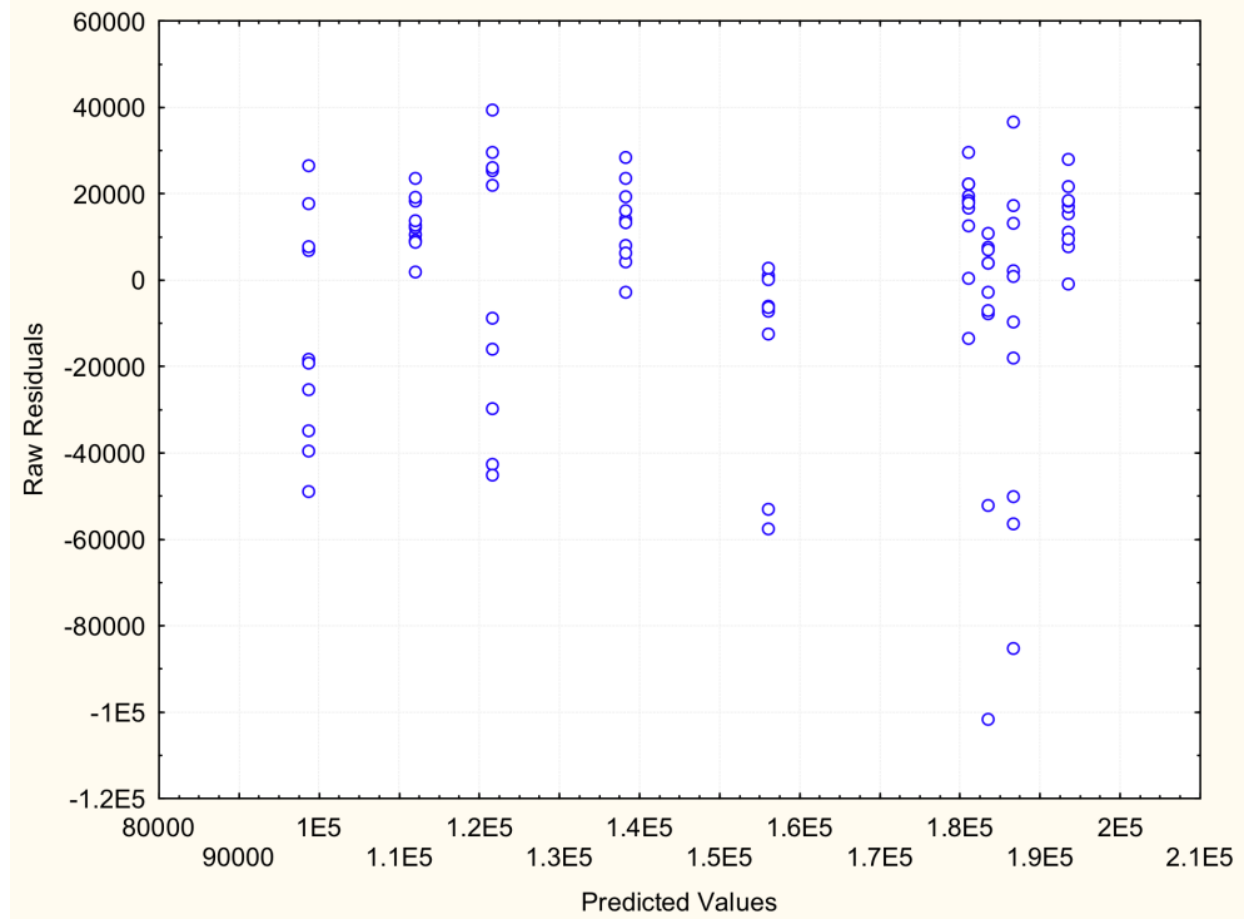

Figure A.8: A plot of the errors vs. the values predicted by the model for equation (2.4)

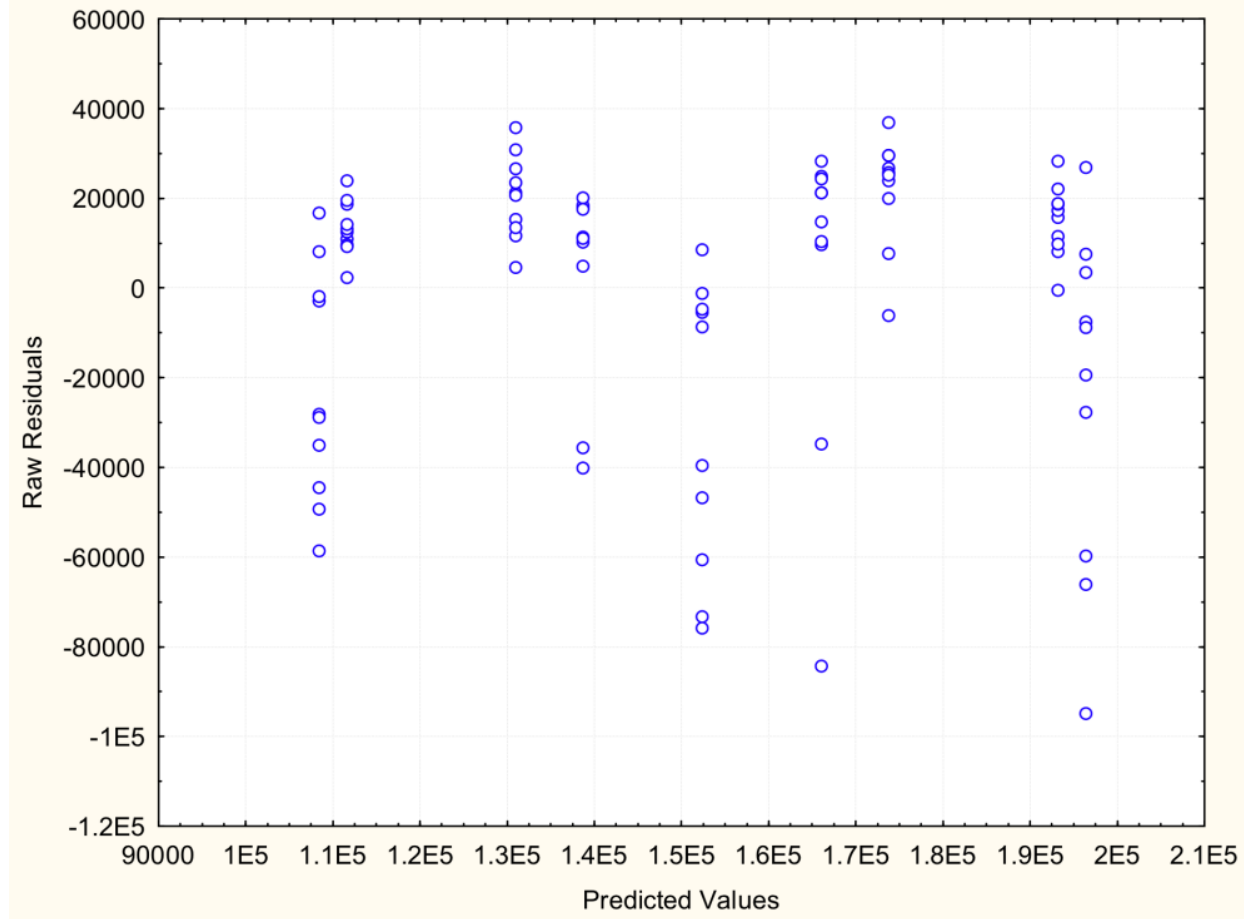

Figure A.9: A plot of the errors vs. the values predicted by the model for equation (2.16) 


\section{A.3 Discussion of Model Assumptions}

The data in Sections A.1 and A.2 shows that the assumptions for the cobalt content model, equation (2.3), are satisfied. This means that the errors in the model represent the random noise within the system, and therefore establishes confidence that the quadratic representation of the data is a reasonable approximation.

For the transverse rupture strength models, the scatter in the experimental data is large, varies between groups, and does not follow a normal distribution. This is expected because tensile strength measurements in brittle materials are subject to a Weibull distribution [2], which is typically skewed to the left for time-to-failure data. The main problem caused by non-normality in the errors is that equations (2.4) and (2.16) tend to under-predict the average value. The data also shows signs of non-constant variance. The main problem this causes is that it tends to overestimate the curvature in the pressure term as discussed previously in Section 2.4. Finally, the fact that the errors do not have a mean of zero is evidence that the data (1) follows a Weibull distribution that is skewed right (see Figure A.5 and A.6), and (2) that the model will under-predict the true mean value. The bias in the model was reduced by simplifying the model of equation (2.4) down to the model of equation (2.16). Overall, the best thing to do to improve the accuracy of equation (2.16) is to reduce the amount of variation in the measurements. 